\title{
George Sudarshan: Perspectives and Legacy
}

\author{
Gopalakrishnan Bhamathi \\ 12216 Antoinette Place, Austin, Texas, USA. E-mail:bhamathig@gmail.com
}

Editors: Vinayak Jagadish \& Danko D. Georgiev

Article history: Submitted on November 29, 2021; Accepted on December 20, 2021; Published on December 24, 2021.

\section{G}

eorge Sudarshan has been hailed as a titan in physics and as one who has made some of the most significant contributions in several areas of physics. This article is an attempt to highlight the seminal contributions he has made in physics and the significant developments that arose from his work. Quanta 2021; 10: 75-104.

\section{Introduction}

Ennackal Chandy George Sudarshan (September 16, 1931 - May 13, 2018) was an Indian-American theoretical physicist and a professor at the University of Texas at Austin. He has made seminal contributions in a large number of research areas, which are listed in Table1. His research activity spans a time period of six decades and a little more. In 2006 a Symposium on the Seven Science Quests of Sudarshan was held at Austin [1]. It brought out fairly comprehensively the progress made in these seven areas until then, subsequent to Sudarshan's path-breaking contributions. However there have been further developments in these areas as well as Tomography. Sudarshan's contributions in the areas of Foundations of Quantum Mechanics and Scattering theory have also been over a period of at least five decades, while his contributions to Quantum Computation span a couple of decades.

(c) (1) This is an open access article distributed under the terms of the Creative Commons Attribution License CC-BY-3.0, which permits unrestricted use, distribution, and reproduction in any medium, provided the original author and source are credited.

\section{Research Areas}

Sudarshan's seminal contributions and a few of the research areas that they spawned are listed in Table 2. Comments on a few selected topics are given below.

\subsection{V-A theory of weak interactions}

Steven Weinberg declared that the Universal V-A theory of Weak Interaction of Sudarshan and Marshak was the key to the Electro-Weak Unification theory, for which he was awarded the 1979 Nobel Prize along with Abdus Salam and Sheldon Glashow. The latter theory then led to The Standard model in elementary particle physics which has stood the test of time, now for five decades. It may be of interest to note that the V-A ("vector" minus "axial vector") theory was developed in 1957 by Sudarshan as part of his research [2-4] for a Ph. D. degree under the supervision of his mentor Robert Eugene Marshak. The first textbook on Particle Physics was written in 1961 by Marshak and Sudarshan, namely, An Introduction to Elementary Particle Physics [5].

\subsection{Symmetries}

Symmetries in Physics can be grouped into four areas: Classical Dynamics, Optics, Particle Physics and Quantum Mechanics. In Classical Dynamics, the application of Symmetries led to No-Interaction theorems [6,7] and the group theoretic treatment of conventional classical dynamics resulted in his treatise with Mukunda, Classical Dynamics: A Modern Perspective [8,9]. In Optics, the Gaussian and Fourier-Maxwell optics was developed, 
Table 1: Seminal contributions of George Sudarshan.

\begin{tabular}{|l|l|}
\hline Research Topic & Year \\
\hline \hline Symmetries in Physics & 1955 \\
\hline V-A theory of Weak interactions & 1957 \\
\hline Tachyons & 1959,1962 \\
\hline Quantum Mechanics: Foundations & 1961 \\
\hline Scattering Theory & 1961 \\
\hline Open Quantum Systems & 1961,1976 \\
\hline Quantum Optics & 1963 \\
\hline Spin and Statistics & 1968 \\
\hline Physics and Philosophy & 1975 \\
\hline Quantum Zeno Effect & 1977 \\
\hline Tomography & 1980 \\
\hline Quantum Computation & 2002 \\
\hline
\end{tabular}

which in turn led to the development of electron optics. In Particle Physics, the SU(2) Symmetry considerations led to sum rules between masses and magnetic moments of elementary particles [10,11] which when extended to $\mathrm{SU}(3)$ symmetry gave rise to the famous Gell-MannOkubo mass relationship and in Quantum Mechanics the study of geometric phases [12].

\subsection{Spin-Statistics Theorem}

Sudarshan believed strongly (some even dubbed it a "religious" belief) that a Non-Relativistic proof of the SpinStatistics theorem [13,14] should be possible. As early as 1968 he gave the non-relativistic proof in 3-dimensions and later extended it to higher dimensions [13-17]. MooYoung Han who proposed with Yoichiro Nambu the Color symmetry of quarks [18], attributes this to the influence of Sudarshan's belief in the validity of the Spin-Statistics theorem. Earlier Han had worked with Sudarshan as a research associate, which led to No-go theorems in combining internal symmetries with space-time theories.

\subsection{Tachyons}

Sudarshan has been described as an iconoclast in the sense that he did not hesitate to go against conventional wisdom if a fresh approach and a thorough investigation warranted it. It is this spirit that led him to show that the existence of particles traveling faster than light, now called tachyons [19,20], did not violate any fundamental laws of physics. Since tachyons cannot manifest themselves as particles in the laboratory, it has not given rise to many further developments. However, there have been experimental results which claim to have detected tunneling effects faster than light. Tachyons have been exploited with greater success in Science fiction.

\subsection{Quantum Coherence Optics}

Sudarshan's Diagonal Coherent Representation [21] was the first and correct theory to lay the foundation for the entire field of Quantum Optics [22]. This led to the observation of Antibunching and sub-Poisson statistics of photons, Squeezed states, Quantum Teleportation, to name but a few.

\subsection{Quantum Zeno Effect}

The Quantum analogue to the classical proposition of Zeno [23] was first proposed by Baidyanath Misra and Sudarshan. This has led to a vast array of research topics such as Decoherence, dissipation, Quantum Information, Quantum Technologies, Quantum control, etc.

\subsection{Open Quantum Systems}

The foundation for Open Quantum Systems was laid by Sudarshan, P. M. Mathews and Jayaseetha Rau [24], where they introduced the concept of dynamical maps describing the finite time evolution of open evolution of a system of interest interacting with a reservoir. Later on, the continuous time evolution was discussed in [25] which is now known as the GKSL (Gorini-KossakowskiSudarshan-Lindblad) equation.

\subsection{Tomography}

This is a burgeoning field. Sudarshan and his collaborators, mostly from Italy and Spain, have made and continued to make significant contributions on this and related topics [26, 27]. I think that it may be of interest to add probably a little known fact. Sudarshan's first piece of work in Tomography was in Industrial application, namely in the development of CITA (Computerized Industrial Tomographic Analyzers) systems, along with a couple of colleagues in Physics at the University of Texas. They even got a couple of patents [28, 29] for it. However, it was the Radon transform involved in it, that caught his attention and interest, which he later used in physics problems [30].

\subsection{Quantum Mechanics}

Sudarshan's contributions to the Foundations of Quantum Mechanics have spanned a period of five decades or more. In the last ten years of his life he had revisited the problem of Time as a dynamical variable [31], Uncertainty relations [32], CPT theorem [33], Scattering theory and Quantum computation. 
Table 2: Areas of further development and research.

\begin{tabular}{|l|l|}
\hline Research Topic & Developments \\
\hline \hline Symmetries in Physics & Classical Dynamics: No interaction theorems \\
& $\begin{array}{l}\text { Optics: Gaussian, Fourier-Maxwell Optics, Electron Optics } \\
\text { Particle Physics: Masses and magnetic moments sum rules } \\
\text { No-go theorems } \\
\text { Quantum Mechanics: Berry phases }\end{array}$ \\
\hline V-A theory of Weak interactions & Electro-weak unification-Standard model \\
\hline Tachyons & Tunneling (faster than light), Science fiction \\
\hline Quantum Mechanics: Foundations & Time operator, Uncertainty relations, CPT theorem \\
\hline Scattering Theory & Cascade model, Re-arrangement collision processes \\
\hline Open Quantum Systems & Decoherence, Dissipation, Quantum information \\
\hline Quantum Optics & $\begin{array}{l}\text { Diagonal Coherent Representation, antibunching, sub-Poisson statistics, } \\
\text { squeezed states, Quantum teleportation }\end{array}$ \\
\hline Spin and Statistics & Non-relativistic proof, Extension to higher dimensions, Color symmetry \\
\hline Quantum Zeno Effect & Quantum control, Quantum Technologies \\
\hline Tomography & $\begin{array}{l}\text { Computerized Industrial Tomographic Analyzer System (CITA) } \\
\text { Physics Applications }\end{array}$ \\
\hline
\end{tabular}

\subsection{Scattering Theory}

I was fortunate to collaborate with Sudarshan in a few of these investigations [34-36]. We developed the Cascade model [37] to study the multi step decay processes. We used the Friedrich-Lee model. Sudarshan was not very satisfied with the conventional scattering theory using asymptotic states. He preferred using solvable models to analyze those aspects of scattering and decay processes where they were applicable. The Cascade model was then extended to deal with re-arrangement collision processes.

\section{Phillosophy and Legacy}

Sudarshan's approach to Philosophy was in the same spirit as his approach to Science. It was, not to take anything for granted but to go to the crux of the problem and then follow the logical consequences, to the utter end (like "following a sinking star") without being distracted by any extraneous considerations. He believed in investigating thoroughly the consequences of the set of precepts on which the system is based. Often he found parallels between physical and philosophical concepts. But he was always careful not to equate the two or assert that one proved the other. Sudarshan was open minded and willing to investigate anything that interested him or piqued his curiosity.

The legacy of George Sudarshan is:

(1) Being open minded in the pursuit of knowledge.

(2) Investigating thoroughly the relevant fundamental principles without any external bias and follow through the consequences to their logical end.
(3) Pursuing one's vocation irrespective of any material rewards; the pursuit and the knowledge gained are in themselves the rewards.

\section{Conclusion}

Finally no description about George Sudarshan's contribution will be complete without a quotation or recitation of a Sanskrit verse. Given below is his favorite verse from the Sri Dakshinamurthy Stotram. This verse exemplifies in a sense the complete circle of his quest. If in physics his quest may be described as "from Being to Becoming" the circle was completed in his philosophical quest "from Becoming to Being".

viśvaṃ darpaṇadṛśyamānanagaritulyạ̣ nijāntargatạ̣ paśyannātmani māyayā bahirivodbhūtam yathā nidrayā. yah sākṣātkurute prabodhasamaye svātmānamevādvayạ̣ tasmai śrígurumūrtaye nama idạ̣ śrídakṣiṇāmūrtaye

The meaning of the Sanskrit verse is:

Similar to the image of a town as seen in the mirror, When one sees the image of the world within him, The world appears as if it is outside. It is similar to his seeing due to illusion During the state of sleep. That the one real fact appears as many different truths, And he realizes, when he wakes up and sees the reality, That he is really the one and only one Atman, Salutation to the God facing the south, Who is the greatest teacher. 


\section{Anecdote}

Sudarshan's first version of "Particles traveling Faster than Light" with his co-author V. K. Deshpande was sent to Physical Review for publication in 1958. There were three referees; the first one said it was incorrect, the second one said it was correct but well known; when Sudarshan requested a third referee, the third one said he had read the manuscript and he agreed with both the referees! Later he rewrote the manuscript with one more author O. M. P. Bilaniuk and got it published in the American Journal of Physics [19].

\section{Acknowledgements}

I wish to thank Vinayak Jagadish, Francesco Petruccione and Karol Życzkowski for inviting me to contribute to the Online Workshop celebrating the 60th anniversary of the paper on dynamical maps authored by Sudarshan, Mathews and Rau [24], organized by the National Institute for Theoretical and Computational Sciences (NITheCS), South Africa and the Institute of Theoretical Physics, Jagiellonian University, Poland on October 14-15, 2021.

\section{References}

[1] Friends of George Sudarshan. Seven Science Quests. The University of Texas at Austin, Austin, Texas, 2006. http://quest.ph.utexas.edu/index. html.

[2] E. C. G. Sudarshan, R. E. Marshak. The nature of the four-fermion interaction. in: N. Zanichelli (Ed.), International Conference on mesons and recently discovered particles e 43e Congresso nazionale di fisica: communicazioni, Padova-Venezia, 22-28 Settembre 1957. Societa Italiana di Fisica, Padova, 1958.

[3] E. C. G. Sudarshan, R. E. Marshak. The nature of the four-fermion interaction. in: P. K. Kabir (Ed.), The Development of Weak Interaction Theory. Gordon and Breach, New York, 1963. pp. 119-128.

[4] E. C. G. Sudarshan, R. E. Marshak. The nature of the four-fermion interaction. Current Science 1992; 63(2):65-75. JSTOR: 24095422.

[5] R. E. Marshak, E. C. G. Sudarshan. Introduction to Elementary Particle Physics. Vol. 11 of Interscience Tracts on Physics and Astronomy. Interscience Publishers, New York, 1961.
[6] D. G. Currie, T. F. Jordan, E. C. G. Sudarshan. Relativistic invariance and Hamiltonian theories of interacting particles. Reviews of Modern Physics 1963; 35(2):350-375. doi:10.1103/RevModPhys.35, 350.

[7] G. Marmo, N. Mukunda, E. C. G. Sudarshan. Relativistic particle dynamics-Lagrangian proof of the no-interaction theorem. Physical Review D 1984; 30(10):2110-2116. doi : 10.1103/PhysRevD. 30. 2110

[8] E. C. G. Sudarshan, N. Mukunda. Classical Dynamics: A Modern Perspective. John Wiley \& Sons, New York, 1974.

[9] E. C. G. Sudarshan, N. Mukunda. Classical Dynamics: A Modern Perspective. World Scientific, Singapore, 2015. doi : 10.1142/9751.

[10] R. Marshak, S. Okubo, G. Sudarshan. Consequences of charge independence for the magnetic moments and masses of $\Sigma$ hyperons. Physical Review 1957; 106(3):599-601. doi:10.1103/PhysRev.106. 599.

[11] S. Okubo, R. E. Marshak, E. C. G. Sudarshan. Isotopic spin selection rules and $K_{2}{ }^{0}$ decay. Physical Review Letters 1959; 2(1):12-14. doi:10.1103/ PhysRevLett.2.12.

[12] R. Simon, H. J. Kimble, E. C. G. Sudarshan. Evolving geometric phase and its dynamical manifestation as a frequency shift: an optical experiment. Physical Review Letters 1988; 61(1):19-22. doi: 10.1103/PhysRevLett.61.19.

[13] I. Duck, E. C. G. Sudarshan. Pauli and the SpinStatistics Theorem. World Scientific, Singapore, 1998. doi : 10.1142/3457.

[14] I. Duck, E. C. G. Sudarshan. Toward an understanding of the spin-statistics theorem. American Journal of Physics 1998; 66(4):284-303. doi: $10.1119 / 1.18860$

[15] E. C. G. Sudarshan. The fundamental theorem on the connection between spin and statistics. in: N. Svartholm (Ed.), Elementary Particle Theory: Relativistic Groups and Analyticity. Proceedings of the Eighth Nobel Symposium held May 19-25, 1968 at Aspenäsgarden, Lerum, in the county of Älvsborg, Sweden. Almqvist and Wiksell, Stockholm, 1968. pp. 379-386. 
[16] E. C. G. Sudarshan. The fundamental theorem on the relation between spin and statistics. Proceedings of the Indian Academy of Sciences - Section A 1968; 67(5):284-293. doi : 10.1007/bf03049366.

[17] L. J. Boya, E. C. G. Sudarshan. The spin-statistics theorem in arbitrary dimensions. International Journal of Theoretical Physics 2007; 46(12):3285-3293. doi : 10.1007/s10773-007-9448-5.

[18] M. Y. Han, Y. Nambu. Three-triplet model with double SU(3) symmetry. Physical Review 1965; 139(4B):B1006-B1010. doi : 10.1103/PhysRev. 139. B1006.

[19] O. M. P. Bilaniuk, V. K. Deshpande, E. C. G. Sudarshan. "Meta" relativity. American Journal of Physics 1962; 30(10):718-723. doi:10.1119/1. 1941773.

[20] M. E. Arons, E. C. G. Sudarshan. Lorentz invariance, local field theory, and faster-than-light particles. Physical Review 1968; 173(5):1622-1628. doi:10.1103/PhysRev.173.1622.

[21] E. C. G. Sudarshan. Equivalence of semiclassical and quantum mechanical descriptions of statistical light beams. Physical Review Letters 1963; 10(7):277-279. doi : 10.1103/PhysRevLett . 10. 277.

[22] J. R. Klauder, E. C. G. Sudarshan. Fundamentals of Quantum Optics. W. A. Benjamin, New York, 1968.

[23] B. Misra, E. C. G. Sudarshan. The Zeno's paradox in quantum theory. Journal of Mathematical Physics 1977; 18(4):756-763. doi : 10.1063/1.523304.

[24] E. C. G. Sudarshan, P. M. Mathews, J. Rau. Stochastic dynamics of quantum-mechanical systems. Physical Review 1961; 121(3):920-924. doi : 10.1103/ PhysRev. 121.920

[25] V. Gorini, A. Kossakowski, E. C. G. Sudarshan. Completely positive dynamical semigroups of $N$ level systems. Journal of Mathematical Physics 1976; 17(5):821-825. doi : 10.1063/1.522979.

[26] V. I. Man'ko, G. Marmo, A. Simoni, A. Stern, E. C. G. Sudarshan, F. Ventriglia. On the meaning and interpretation of tomography in abstract Hilbert spaces. Physics Letters A 2006; 351(1-2):112. doi:10.1016/j.physleta.2005.10.063

[27] M. Asorey, P. Facchi, V. I. Man'ko, G. Marmo, S. Pascazio, E. C. G. Sudarshan. Generalized tomographic maps. Physical Review A 2008; 77(4):042115. doi:10.1103/PhysRevA. 77.042115 .
[28] I. L. Morgan, E. C. G. Sudarshan, A. L. Mitchell, J. P. Coose, H. D. Ellinger, J. W. Jagger. Method and apparatus for tomographic examination of an object by penetrating radiation. US Patent 4284895 , 1981. https://patents.google.com/patent/ US4284895A.

[29] I. L. Morgan, E. C. G. Sudarshan, A. L. Mitchell, J. P. Coose, H. D. Ellinger, J. W. Jagger. Method and apparatus for measuring radiation in computerassisted tomography and radiographic applications. US Patent 4437006, 1984. https://patents. google.com/patent/US4437006A.

[30] M. Asorey, P. Facchi, V. I. Man'ko, G. Marmo, S. Pascazio, E. G. C. Sudarshan. Radon transform on the cylinder and tomography of a particle on the circle. Physical Review A 2007; 76(1):012117. doi : 10.1103/PhysRevA.76.012117

[31] G. Bhamathi, E. C. G. Sudarshan. Time as a dynamical variable. Physics Letters A 2003; 317(5):359364. doi:10.1016/j .physleta. 2003.09.007.

[32] E. C. G. Sudarshan, C. B. Chiu, G. Bhamathi. Generalized uncertainty relations and characteristic invariants for the multimode states. Physical Review A 1995; 52(1):43-54. doi : 10. 1103/PhysRevA. 52 . 43.

[33] M. Selover, E. C. G. Sudarshan. Derivation of the TCP theorem using action principles 2013; arXiv: 1308.5110 .

[34] C. B. Chiu, E. C. G. Sudarshan, G. Bhamathi. Hamiltonian model for the Higgs resonance. Physical Review D 1992; 45(3):884-891. doi:10.1103/ PhysRevD.45.884.

[35] E. C. G. Sudarshan, C. B. Chiu, G. Bhamathi. Perturbation theory on generalized quantum mechanical systems. Physica A: Statistical Mechanics and its Applications 1994; 202(3):540-552. doi : 10.1016/0378-4371(94)90478-2.

[36] G. Bhamathi, E. C. G. Sudarshan. Double resonances and Jordan block spectra. International Journal of Modern Physics B 1996; 10(13-14):15311544. doi : 10.1142/SQ217979296000635.

[37] C. B. Chiu, E. C. G. Sudarshan, G. Bhamathi. Cascade model: a solvable field theory. Physical Review D 1992; 46(8):3508-3528. doi:10.1103/ PhysRevD . 46.3508. 


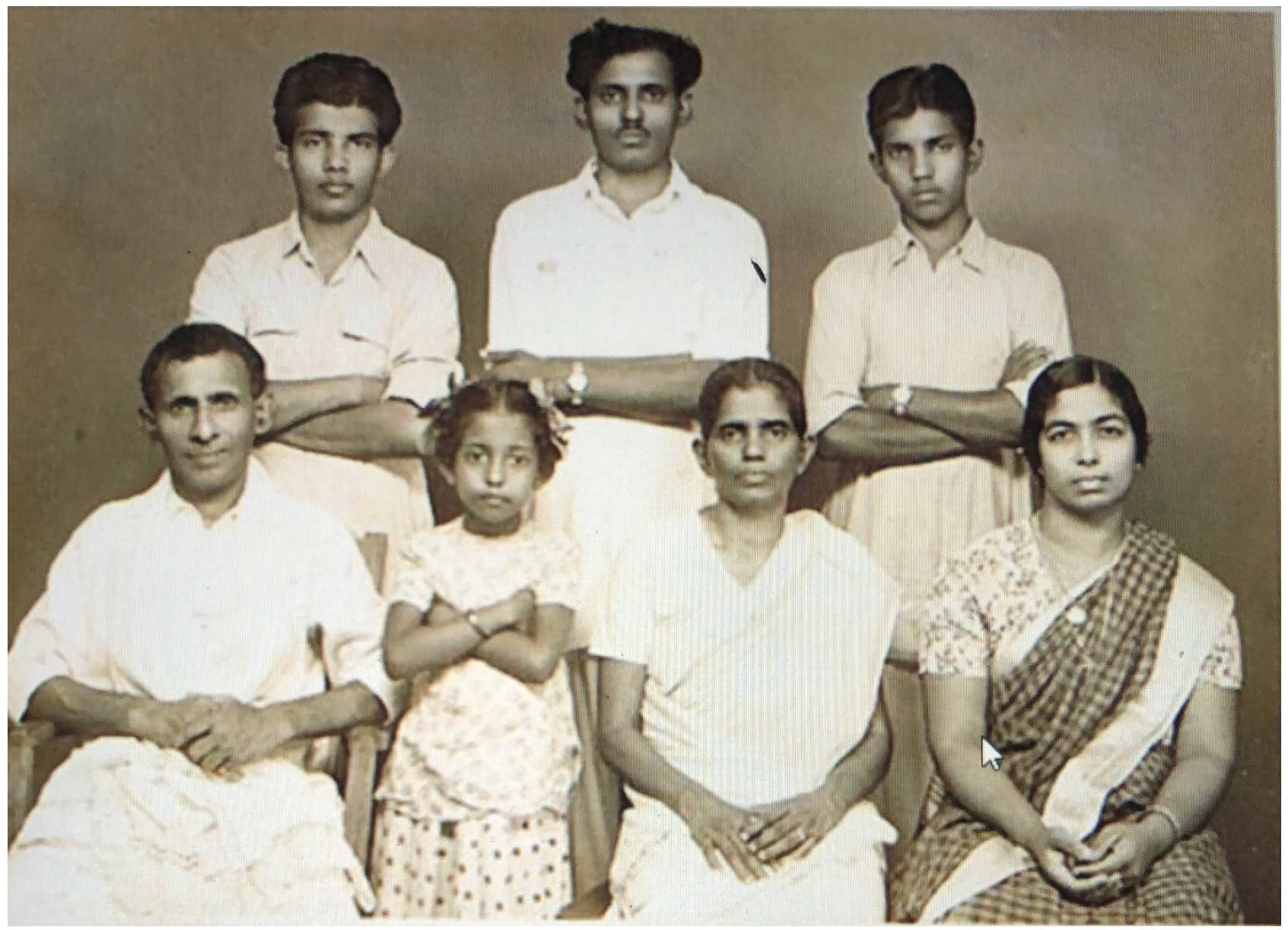

Figure 1: Sudarshan's family. Standing (left to right): George Sudarshan, E. C. Joseph (brother), Thomas Alexander (brother). Sitting (left to right): Ipe Chandy (father), Rajamma (niece), Achamma (mother), Thankamma (sister in law). 


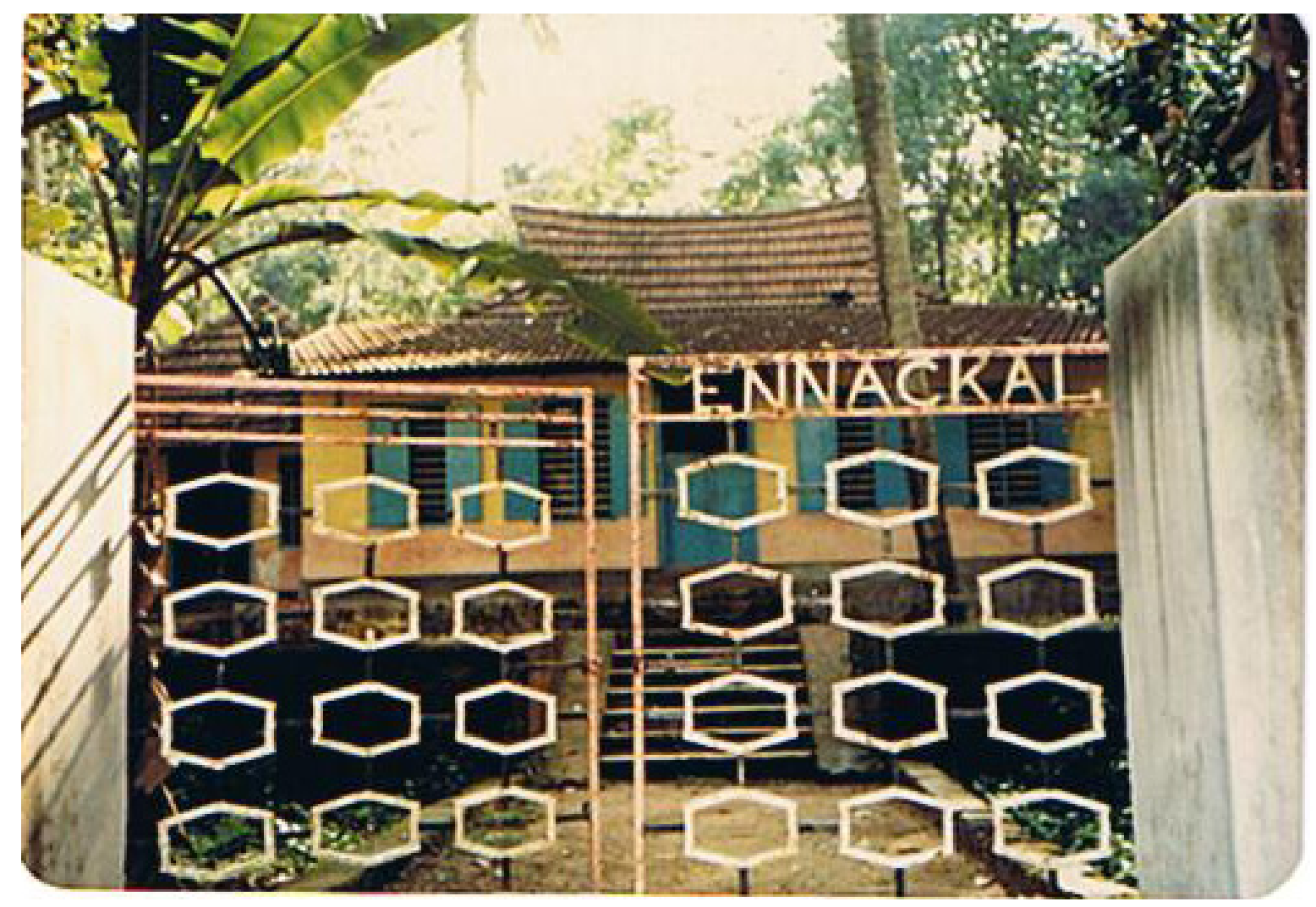

Figure 2: Sudarshan's home.

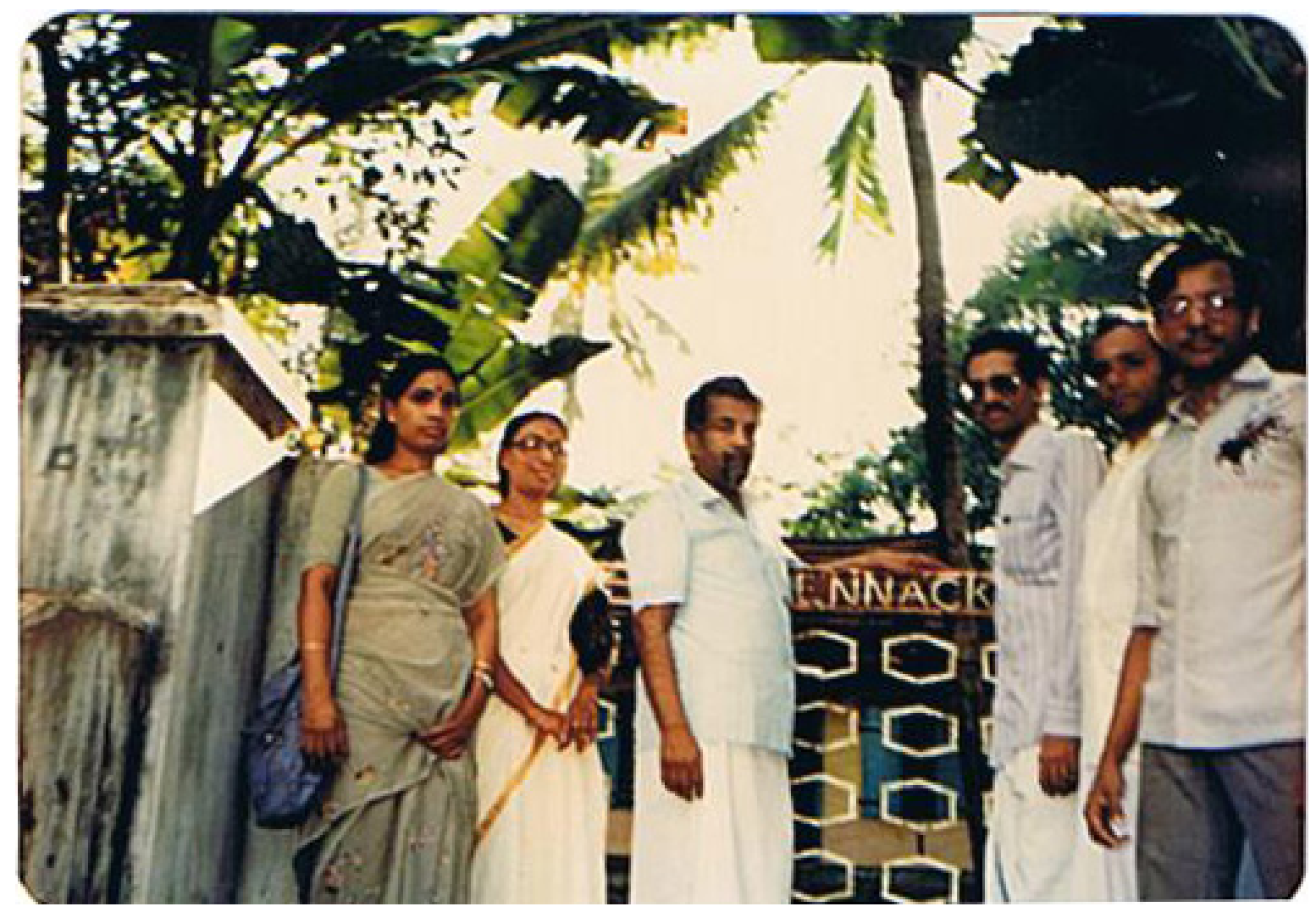

Figure 3: Sudarshan's family and friends. 


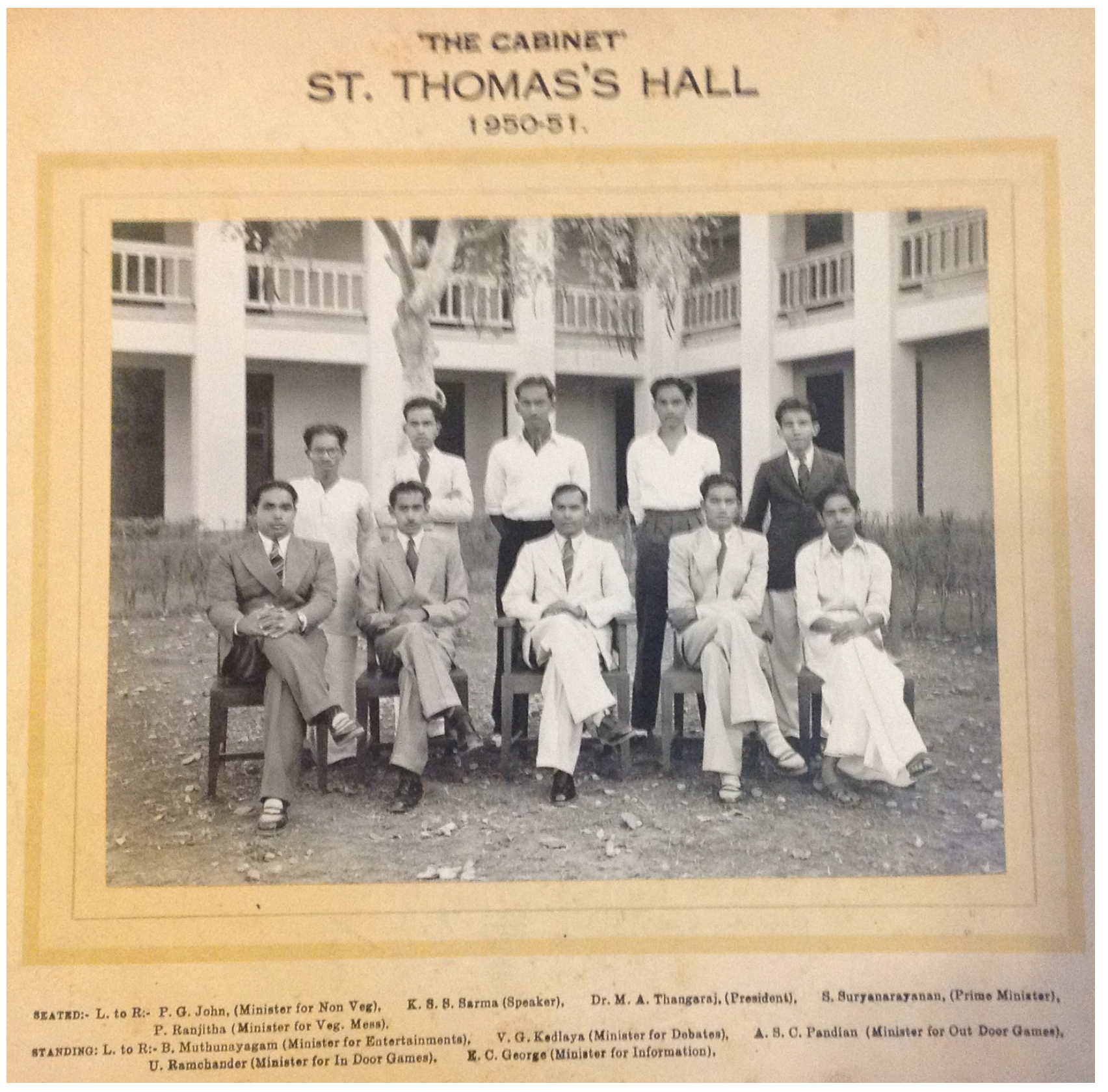

Figure 4: Madras Christian College Batch 1950-1951. 


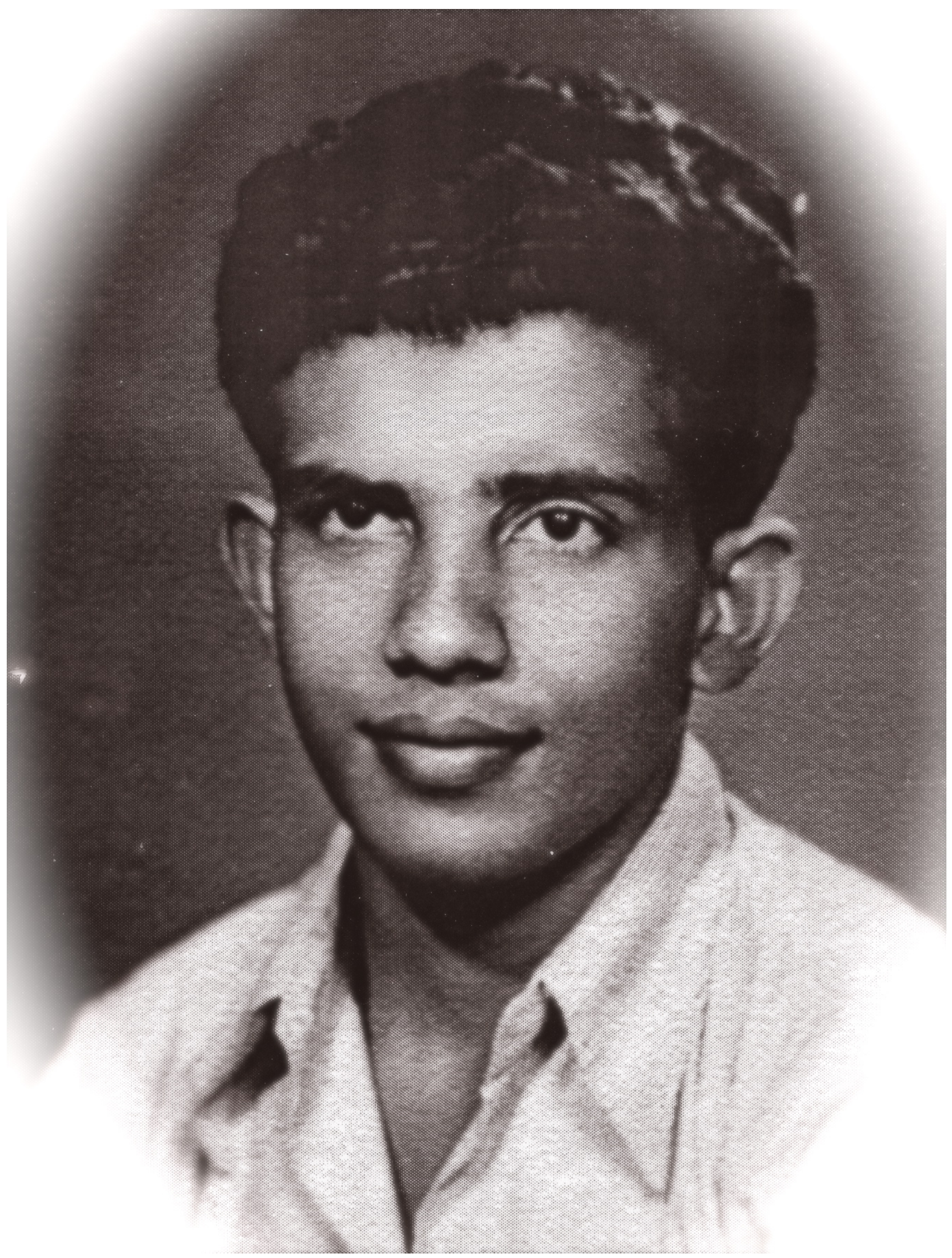

Figure 5: Sudarshan's photograph in Ph.D. Application. 


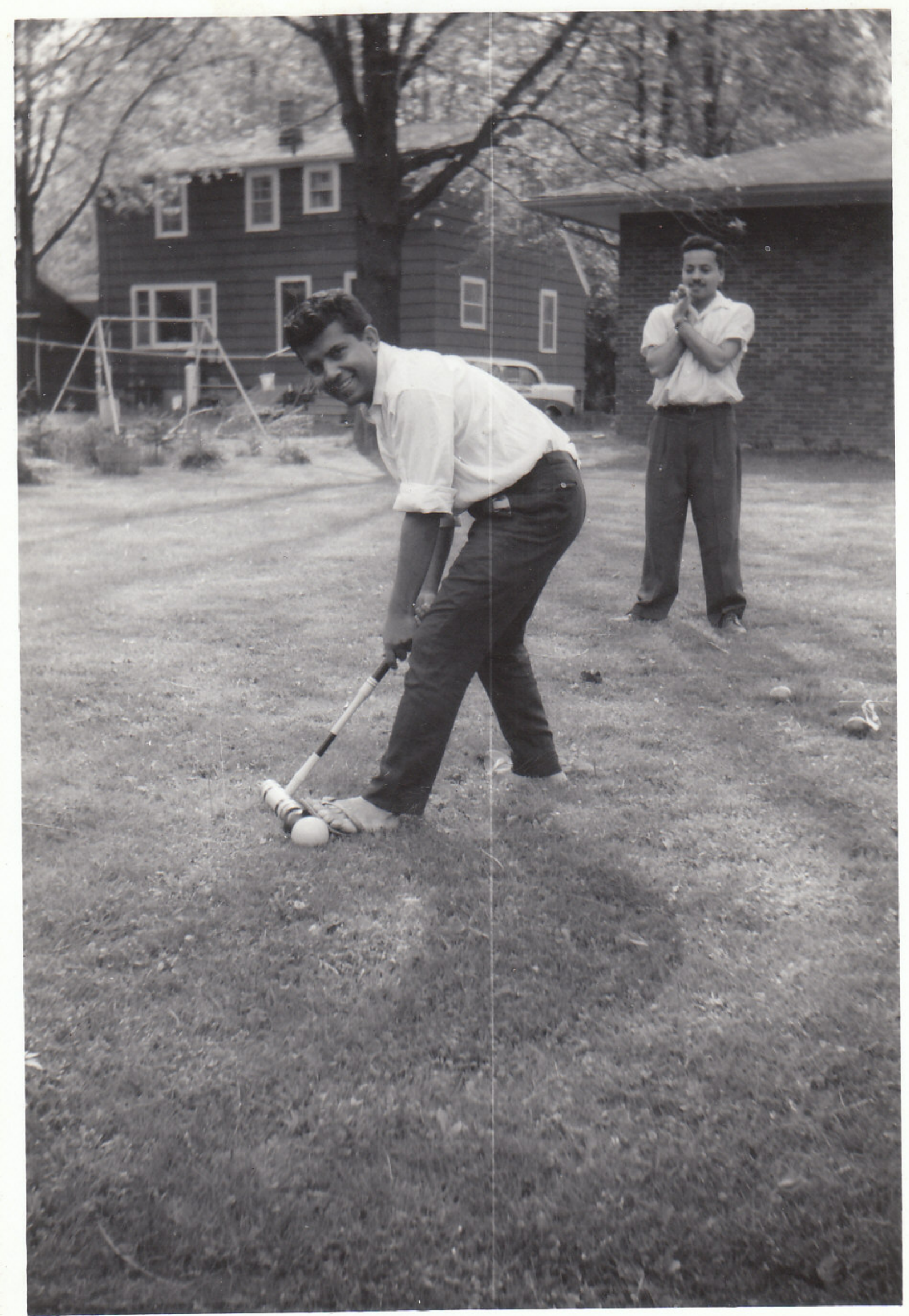

Figure 6: George playing Croquet in Rochester. 


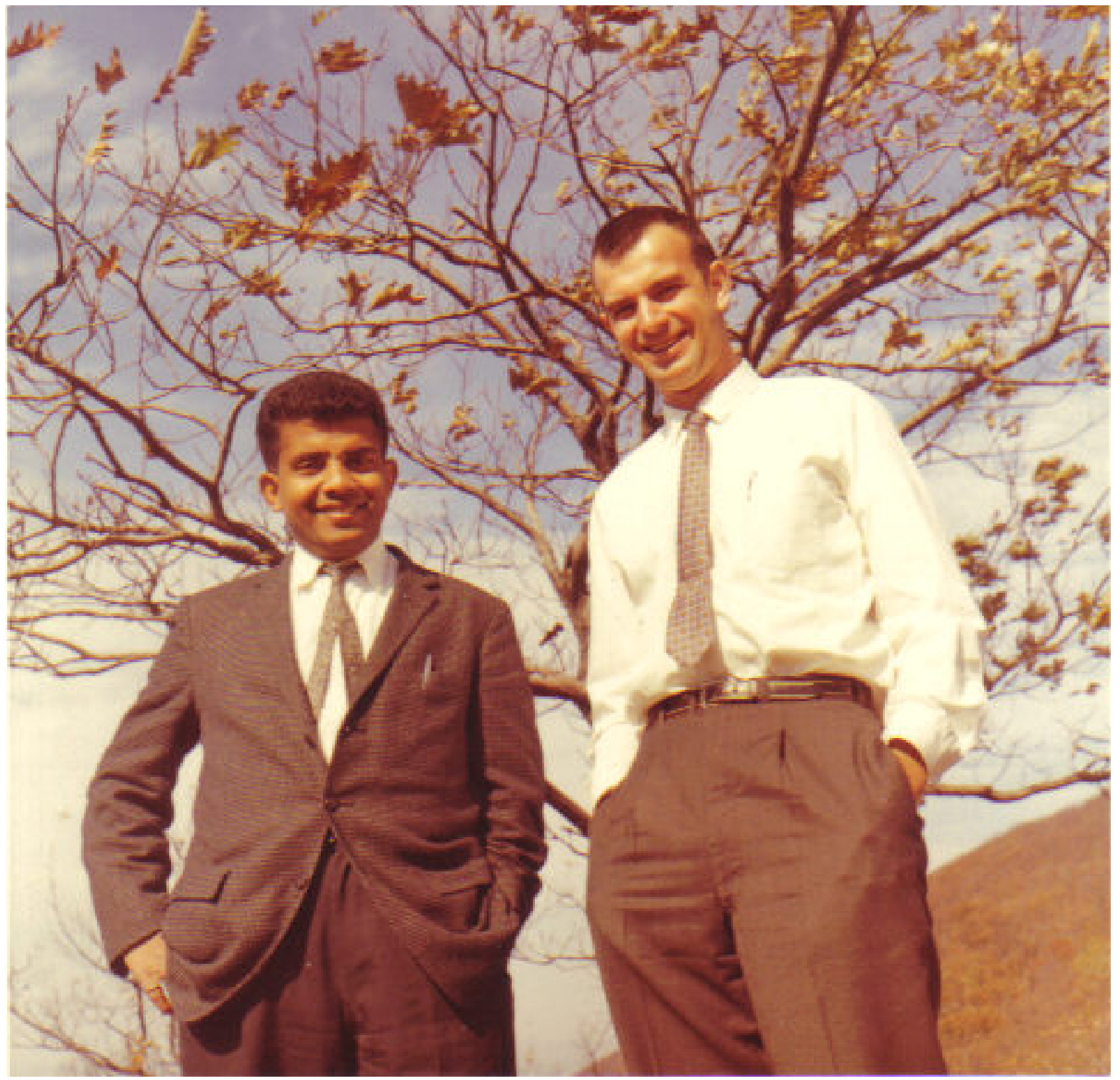

Figure 7: George Sudarshan and Thomas F. Jordan in Rochester. 


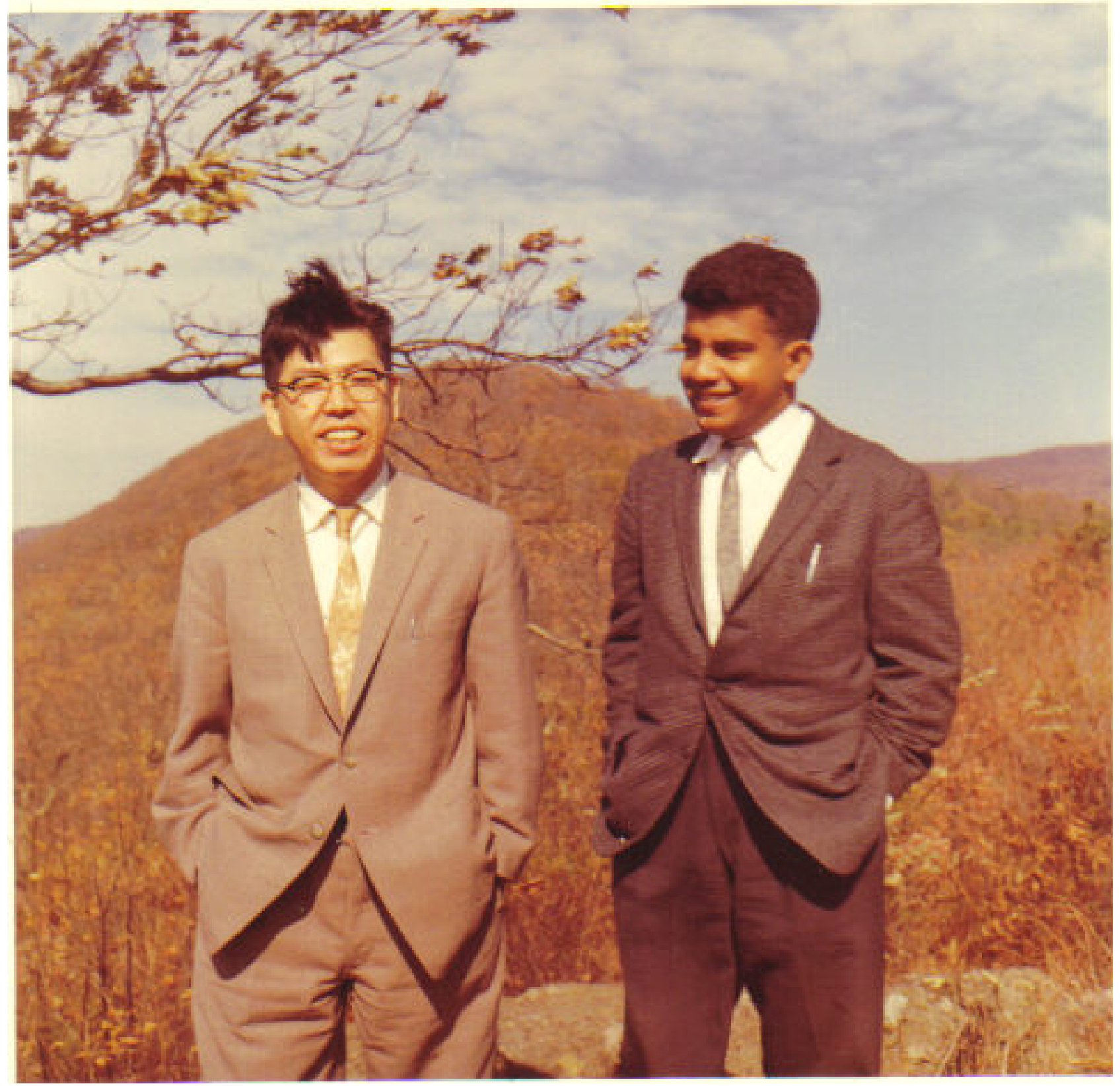

Figure 8: Susumu Okubo and George Sudarshan in Rochester. 


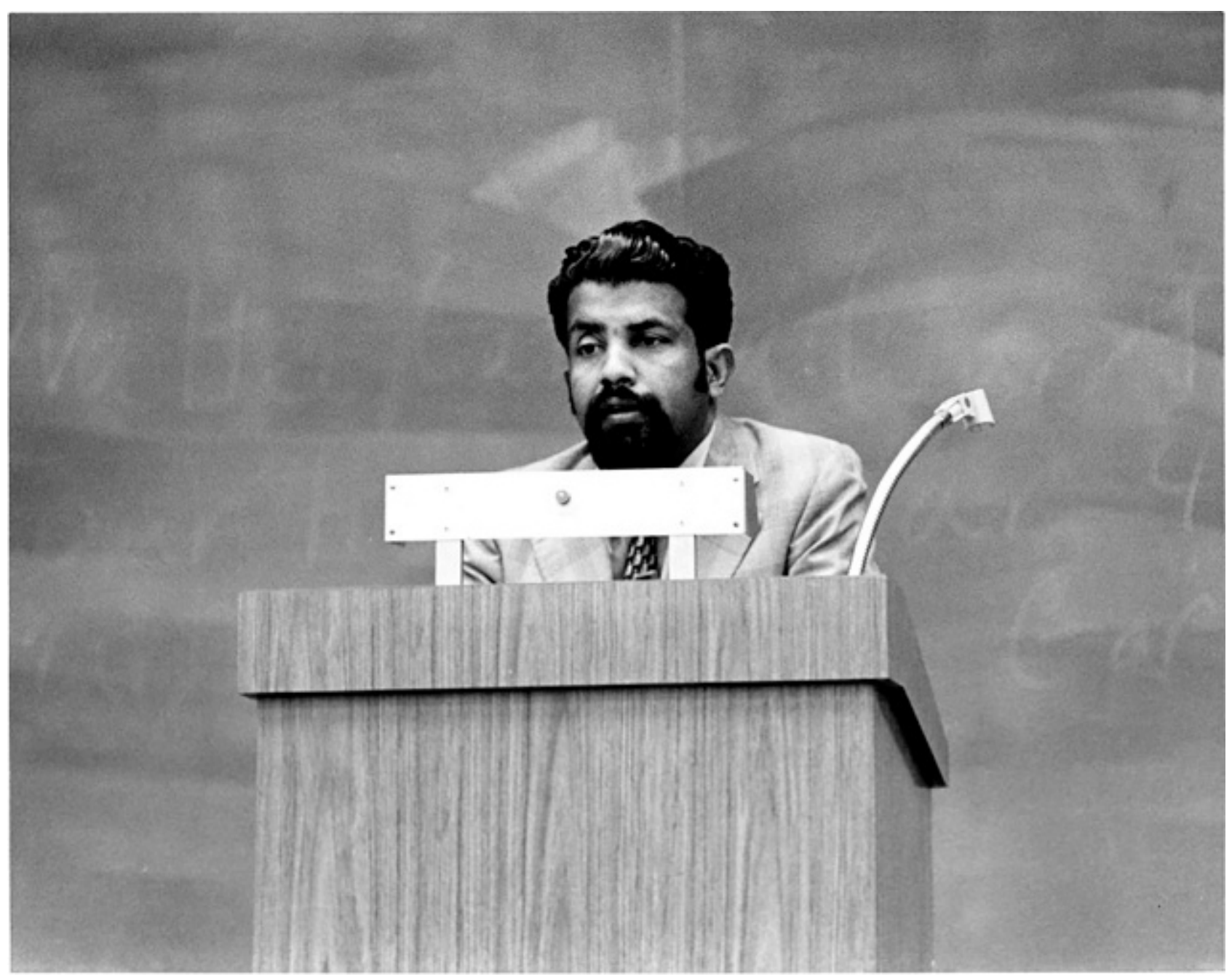

Figure 9: George Sudarshan lecturing at the Austin Conference in 1971. 


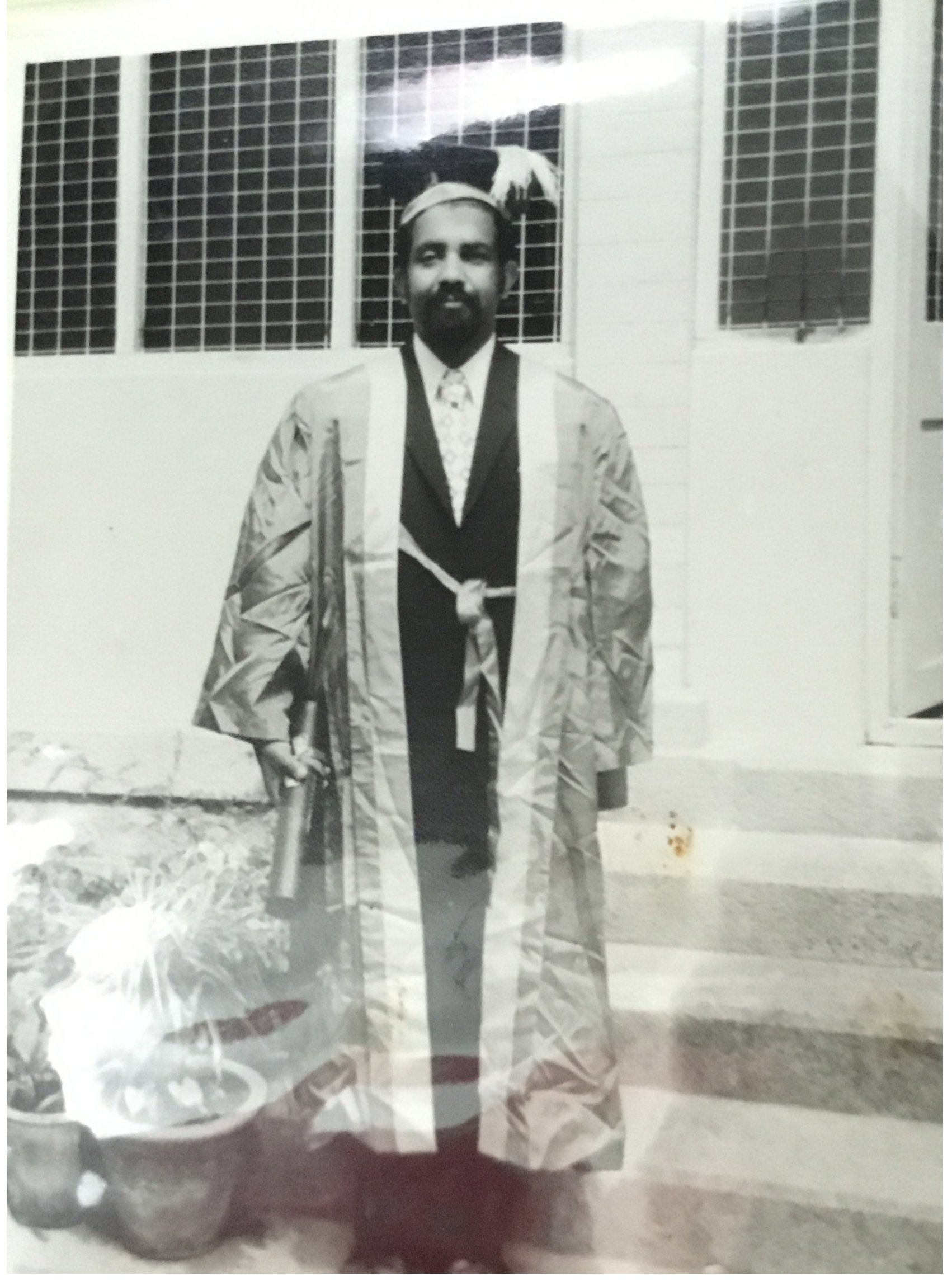

Figure 10: Honorary Doctorate of the Delhi University in 1974. 

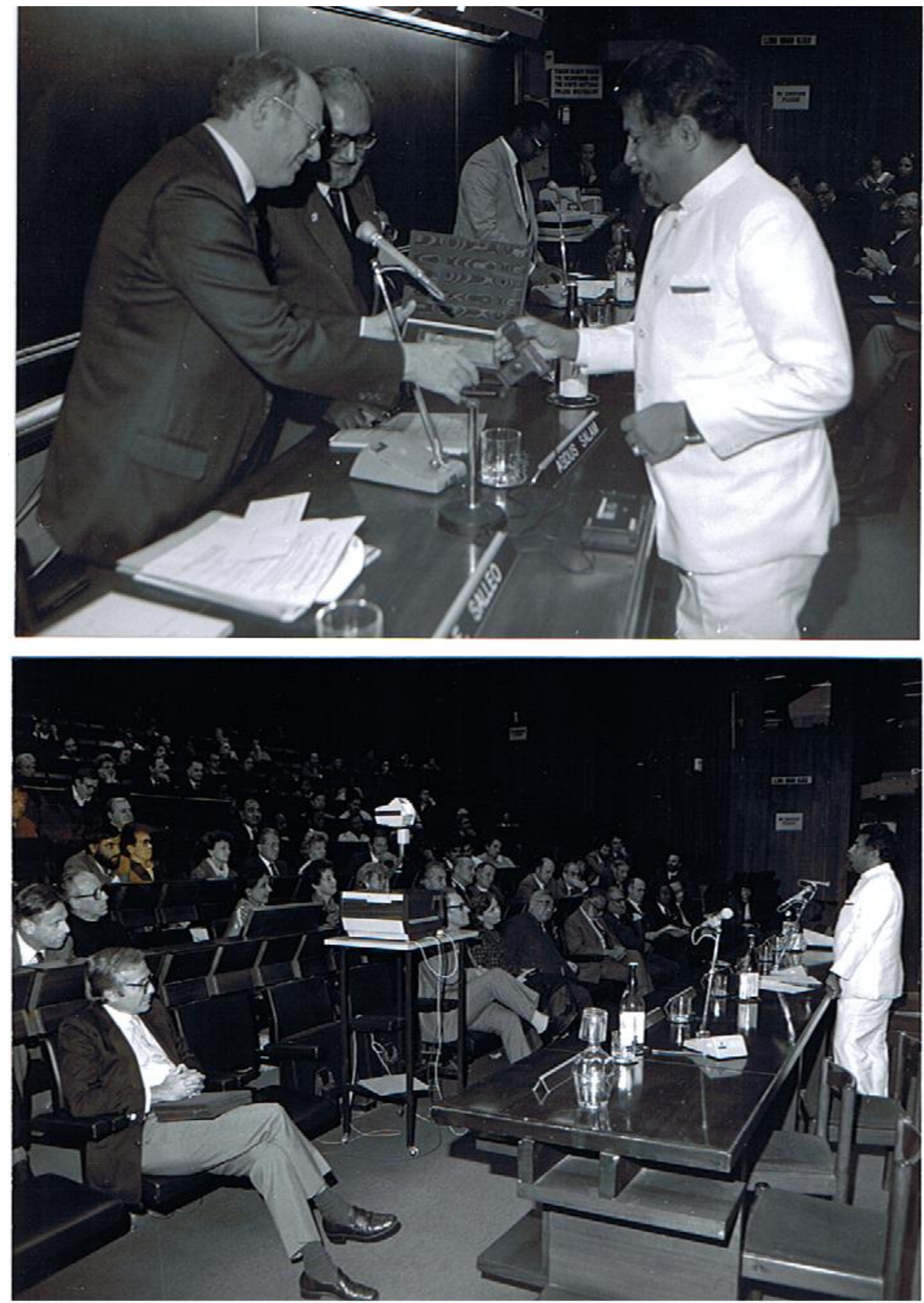

Figure 11: The World Academy of Sciences (TWAS) Prize in 1985. 


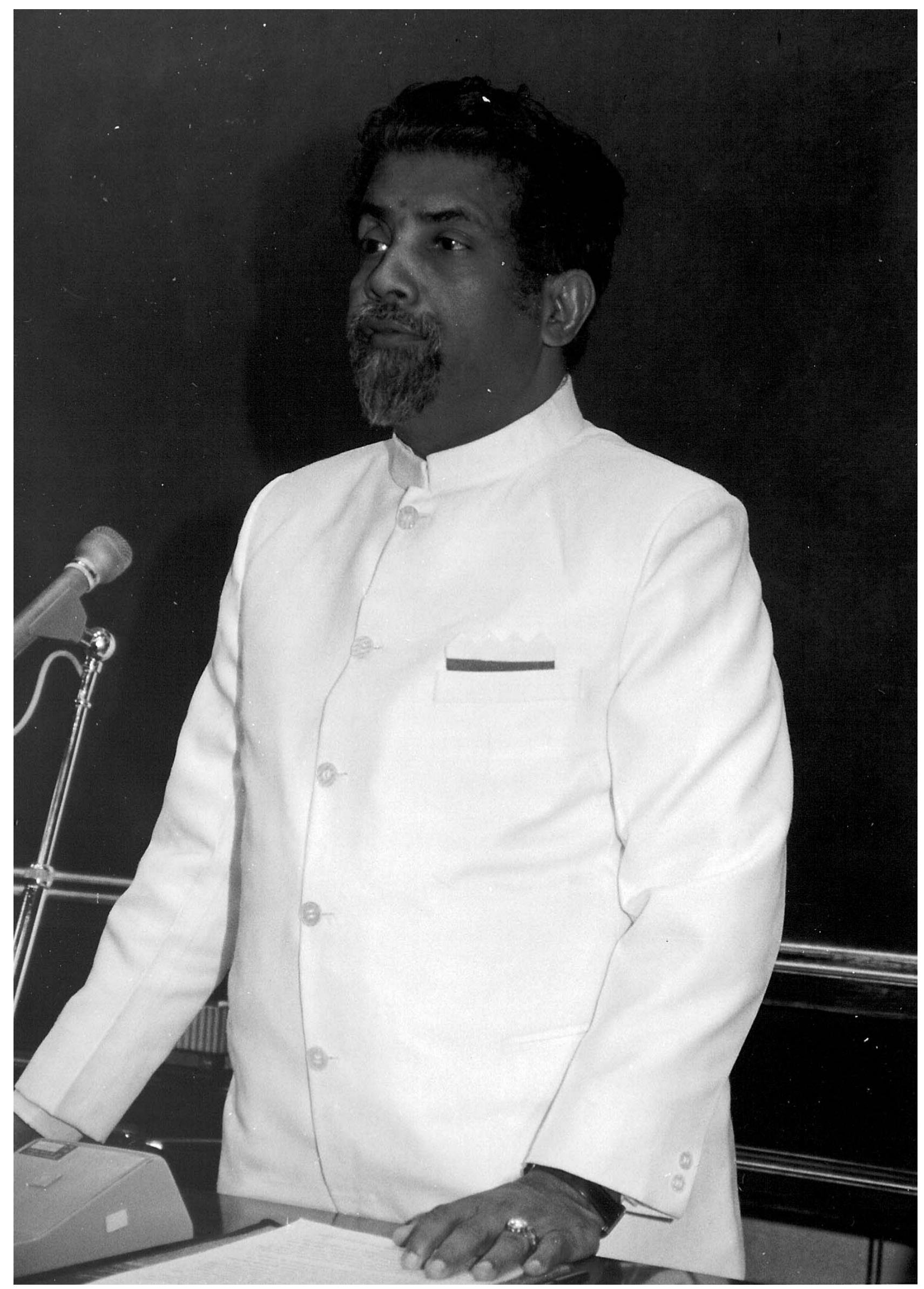

Figure 12: TWAS Award Lecture in 1985. 


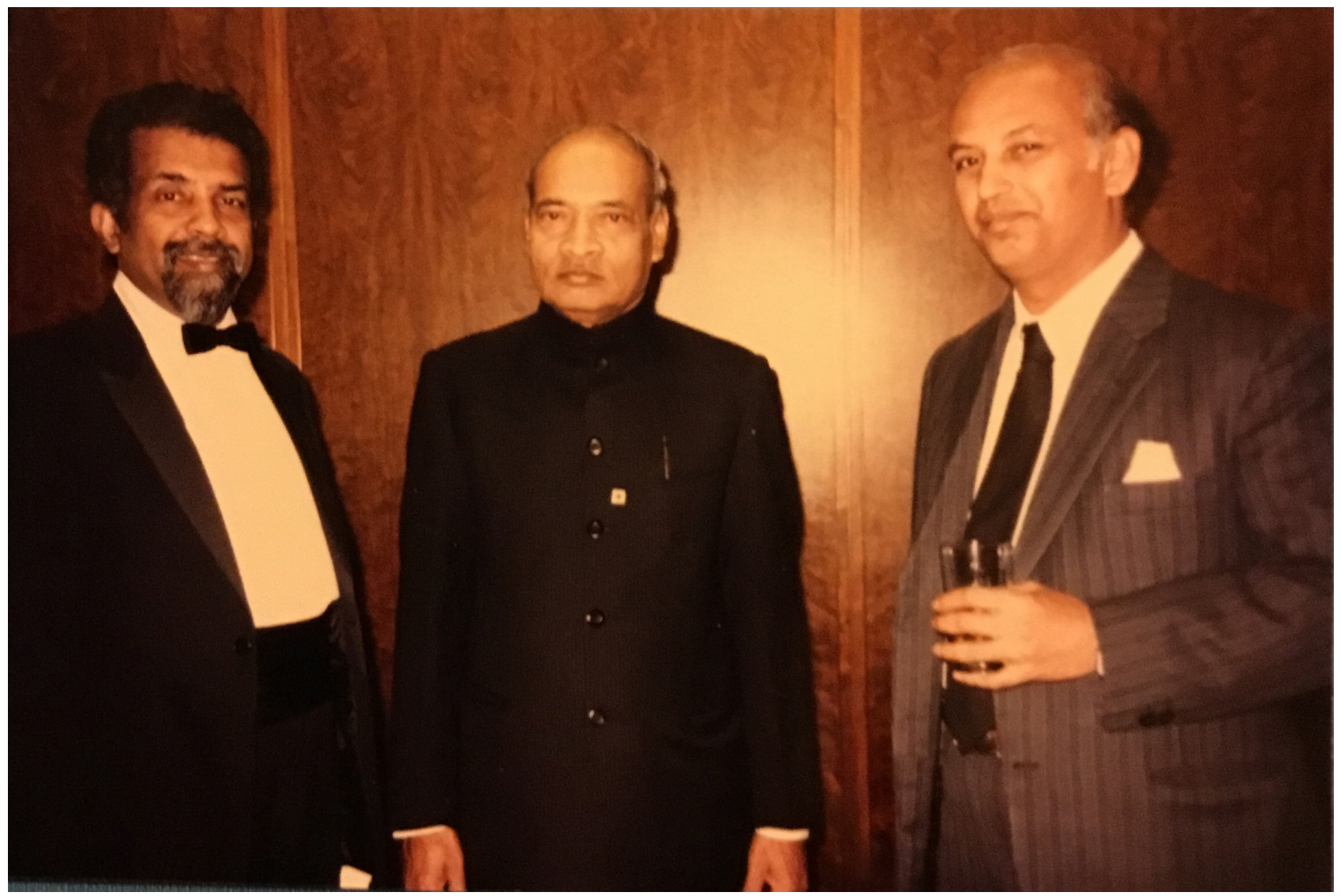

Figure 13: With Narasimha Rao (Former Prime Minister of India) and U. R. Rao in Washington DC. 


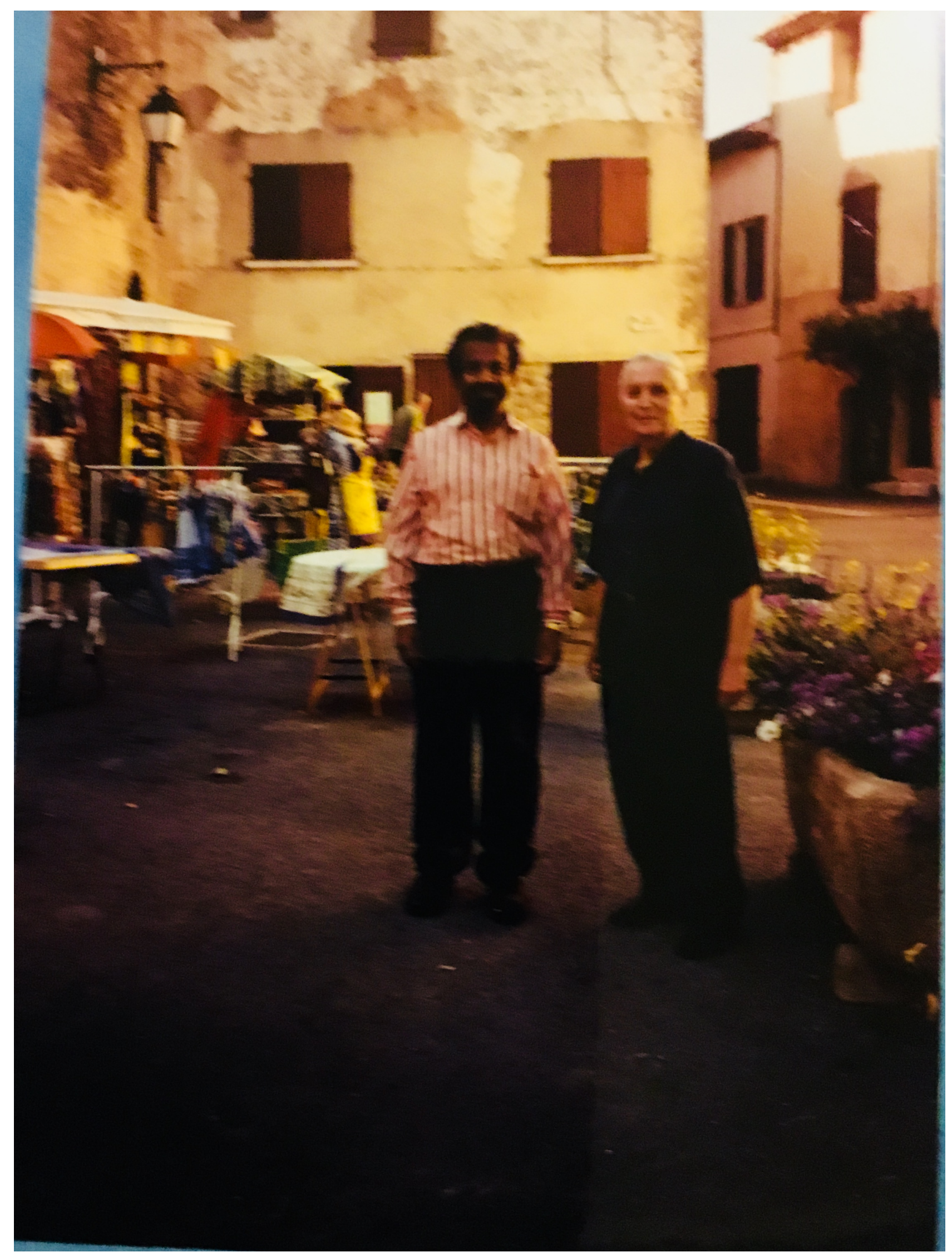

Figure 14: George Sudarshan and Ilya Prigogine, Les Treilles. 


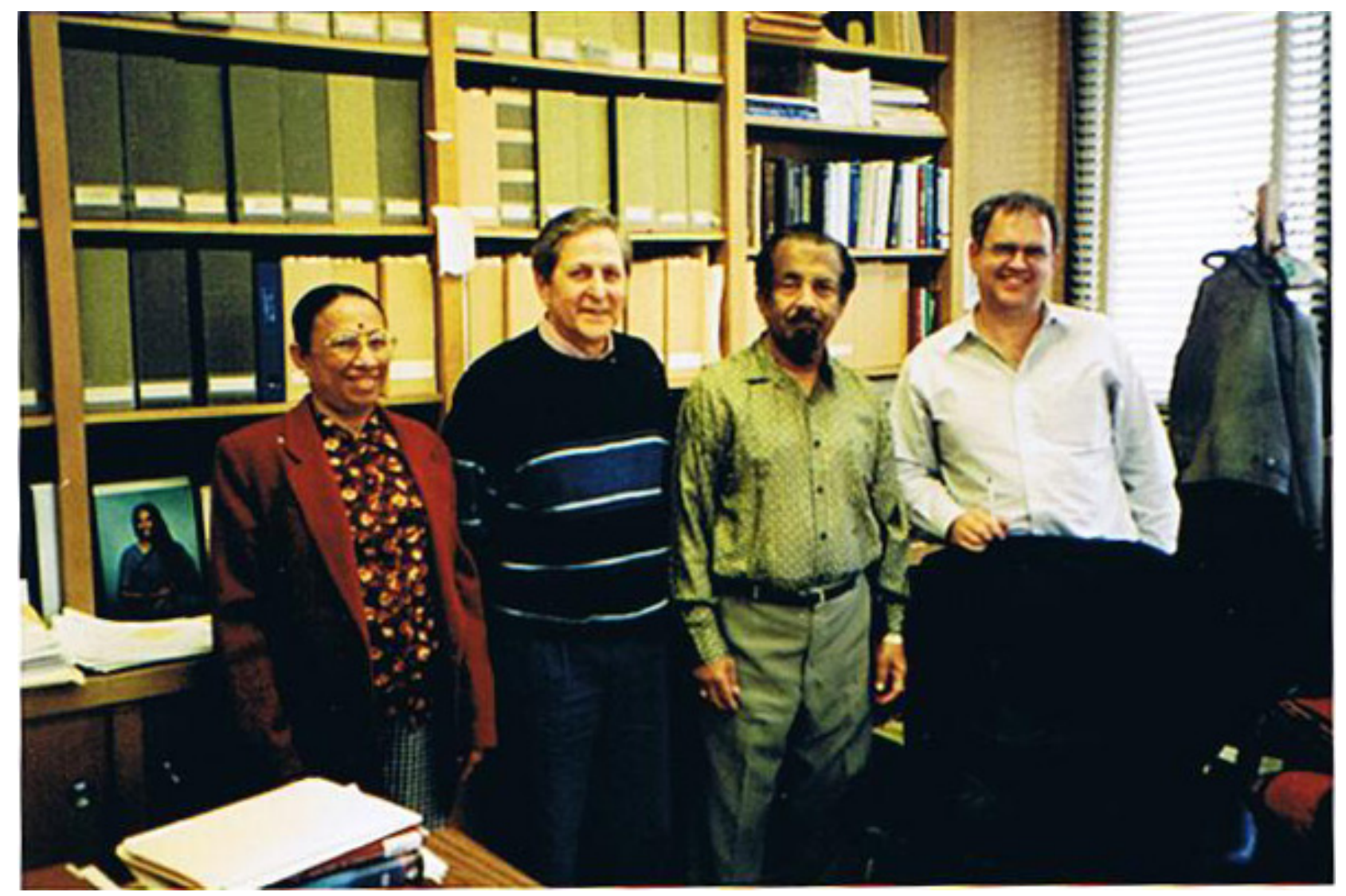

Figure 15: G. Bhamathi, Claude Cohen-Tannoudji, George Sudarshan and Daniel J. Heinzen.

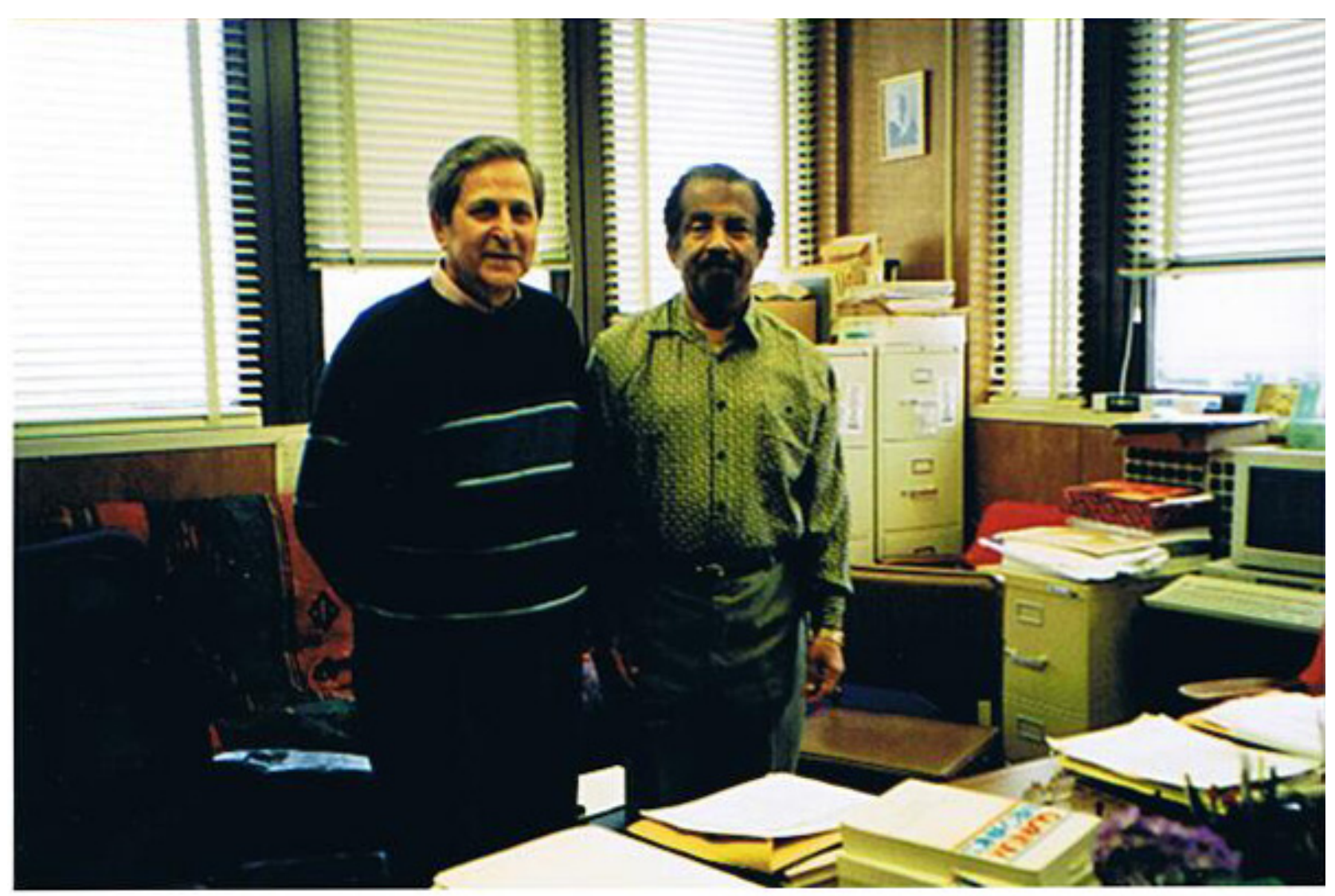

Figure 16: Claude Cohen-Tannoudji and George Sudarshan. 


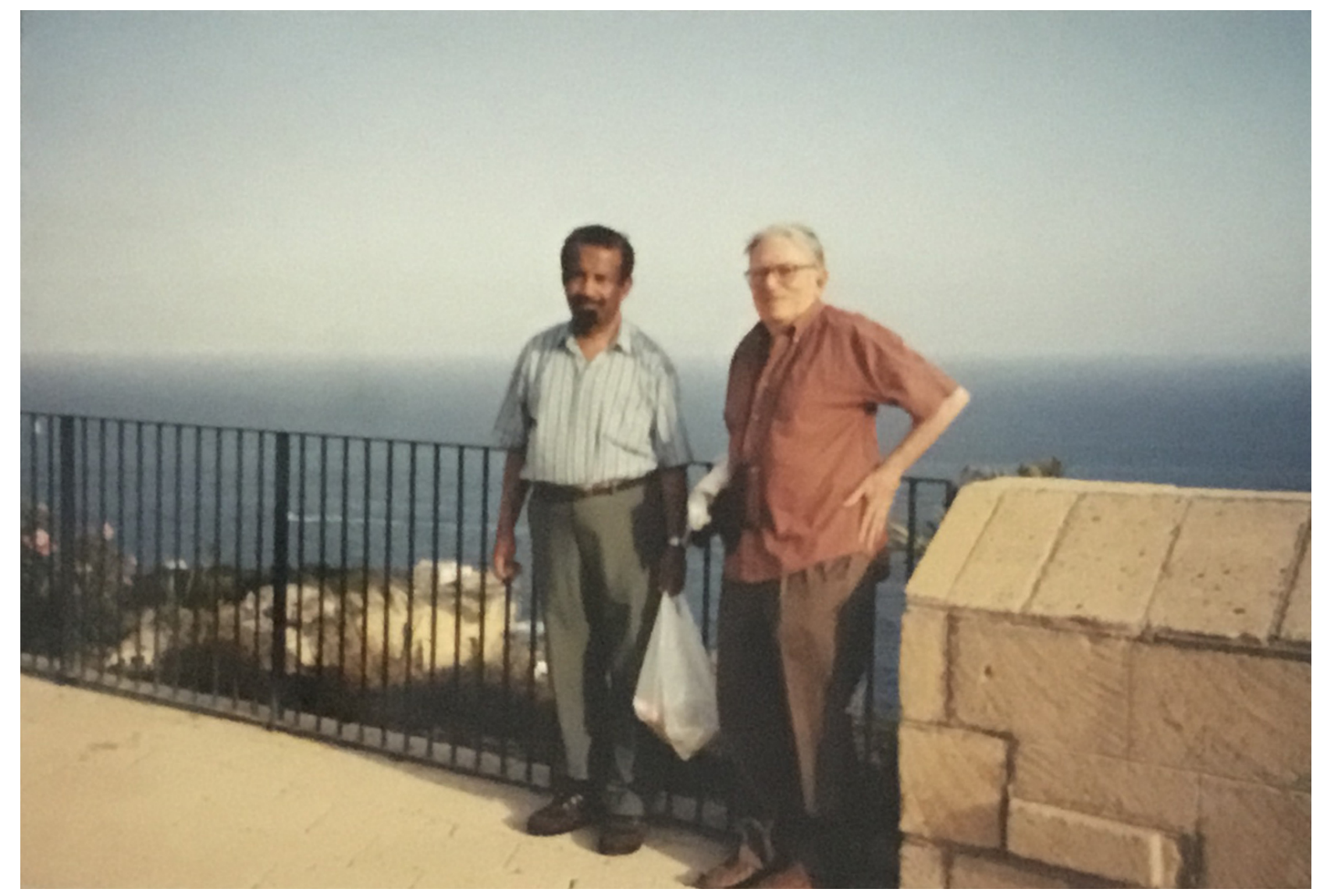

Figure 17: George Sudarshan and Francesco Zaccaria.

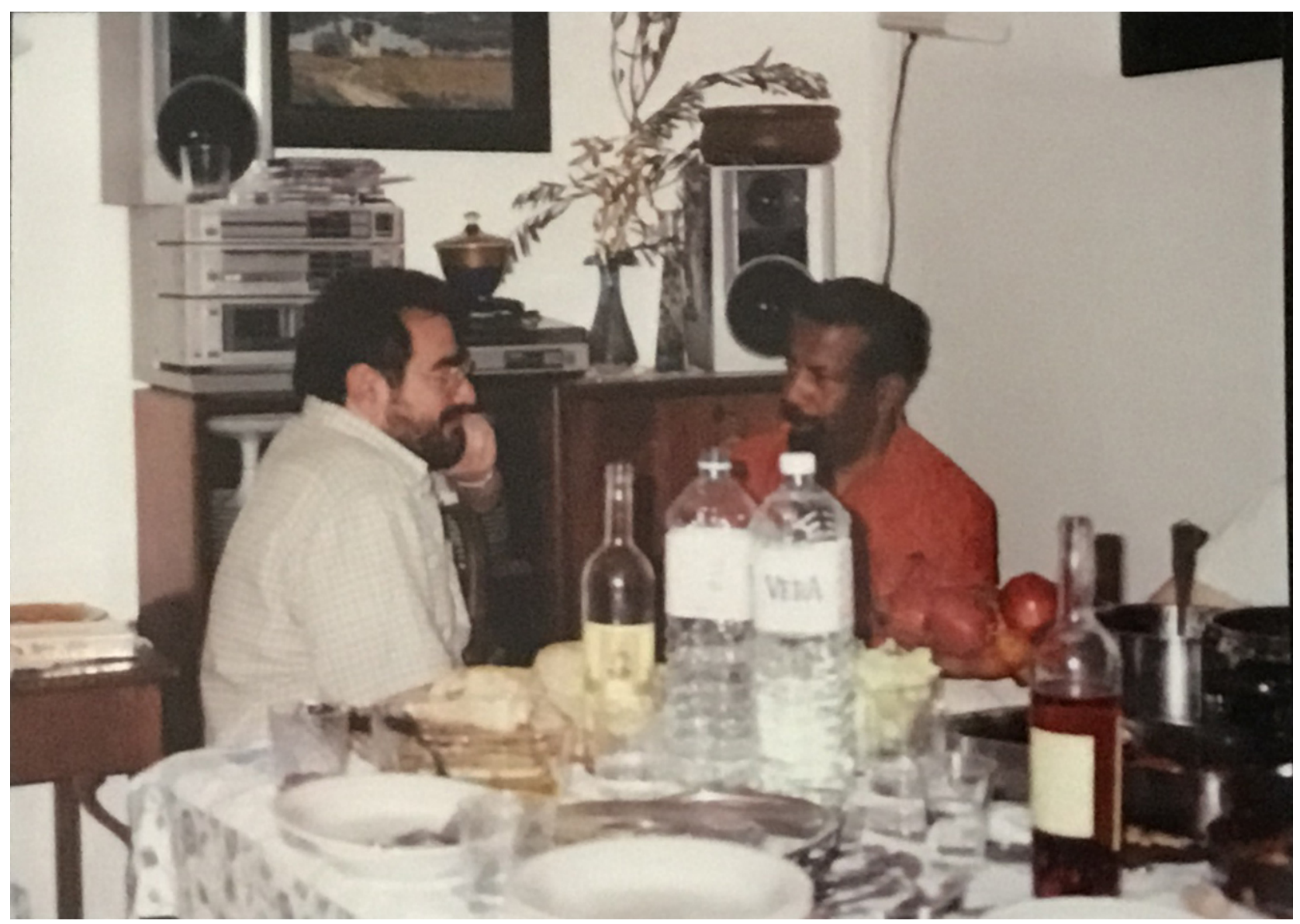

Figure 18: George Sudarshan and Giuseppe Marmo in Napoli. 


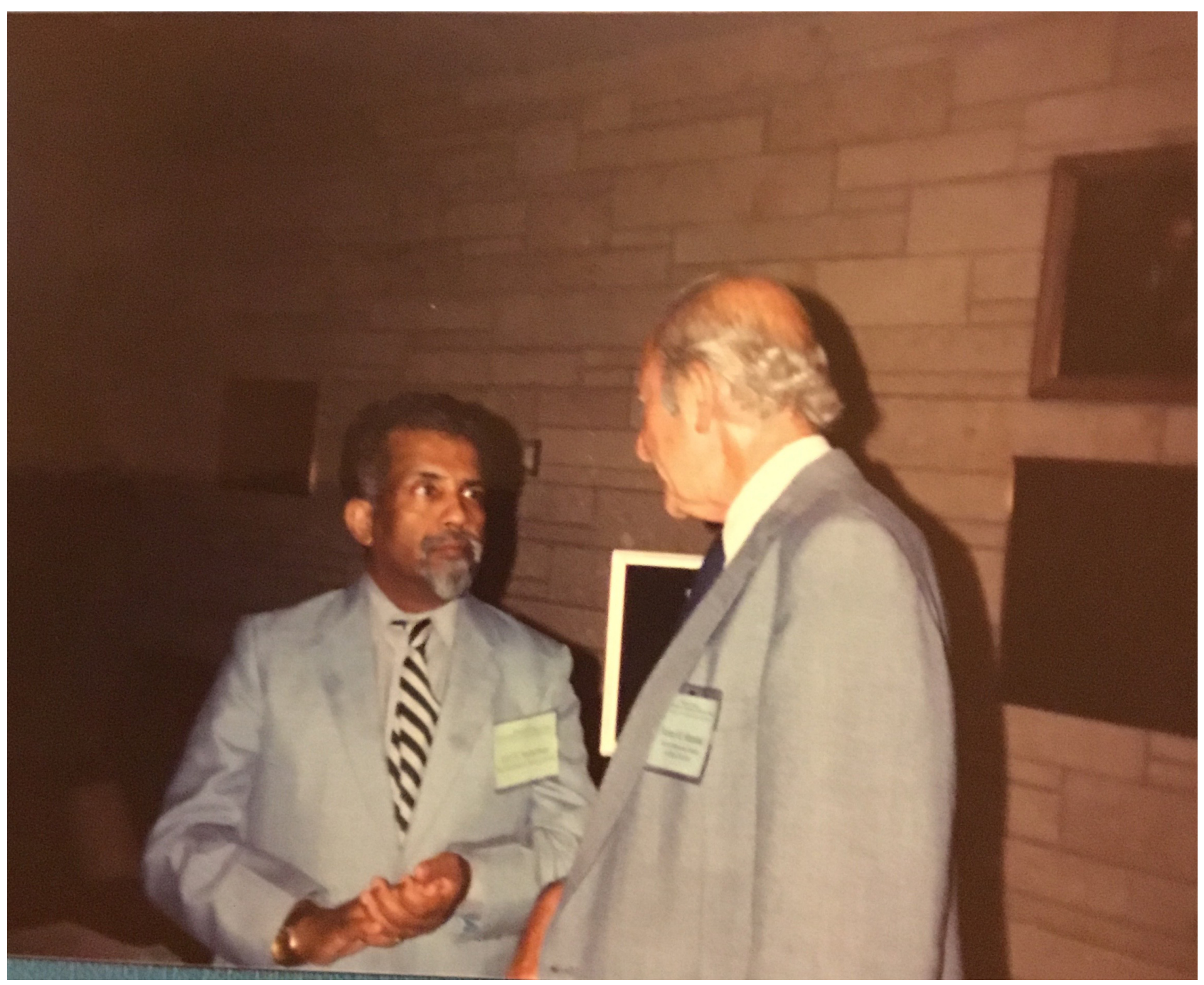

Figure 19: With R. E. Marshak at the 60th Birthday symposium held at Austin in 1991. 


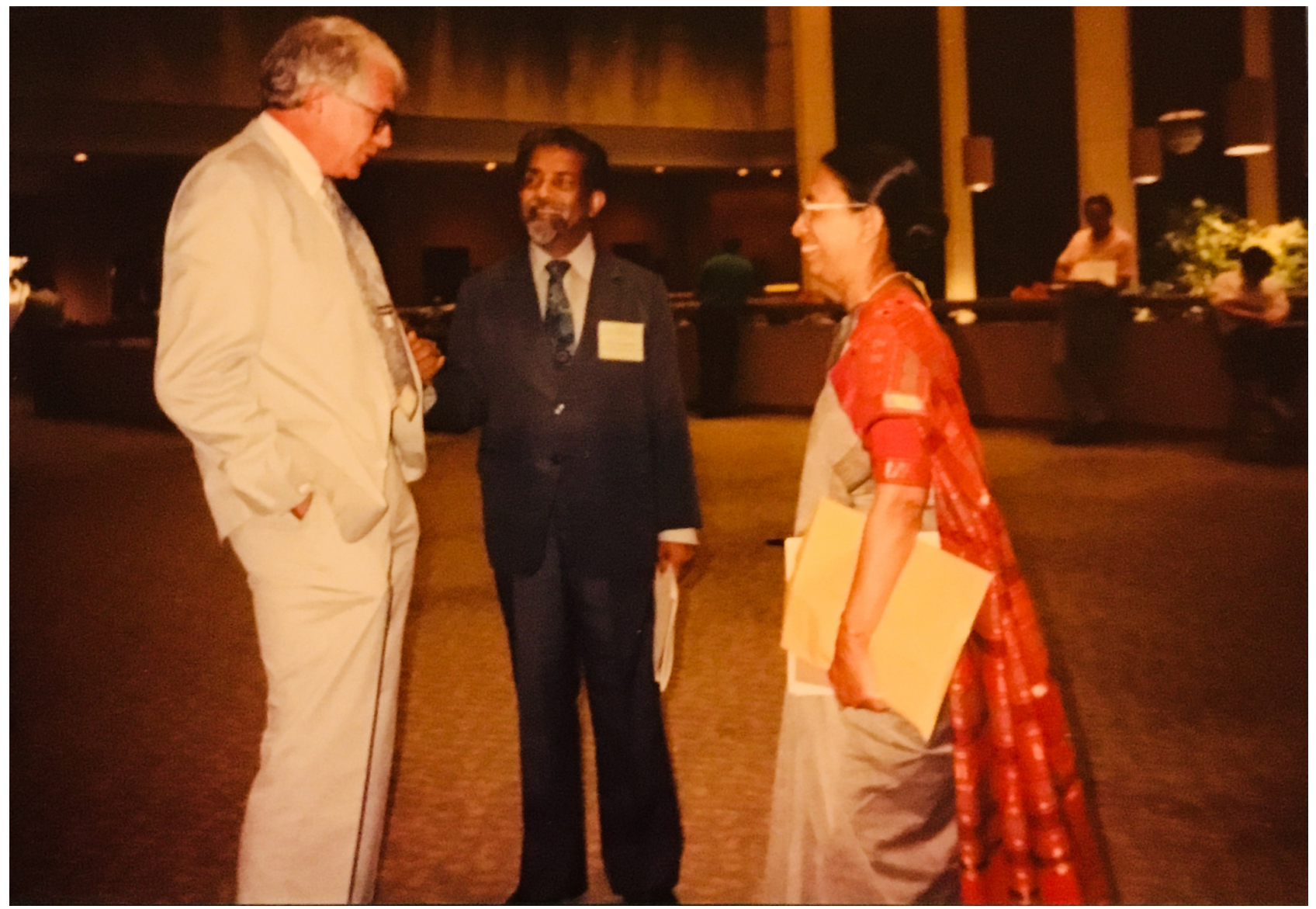

Figure 20: With S. L. Glashow and G. Bhamathi at the 60th Birthday symposium held at Austin in 1991. 

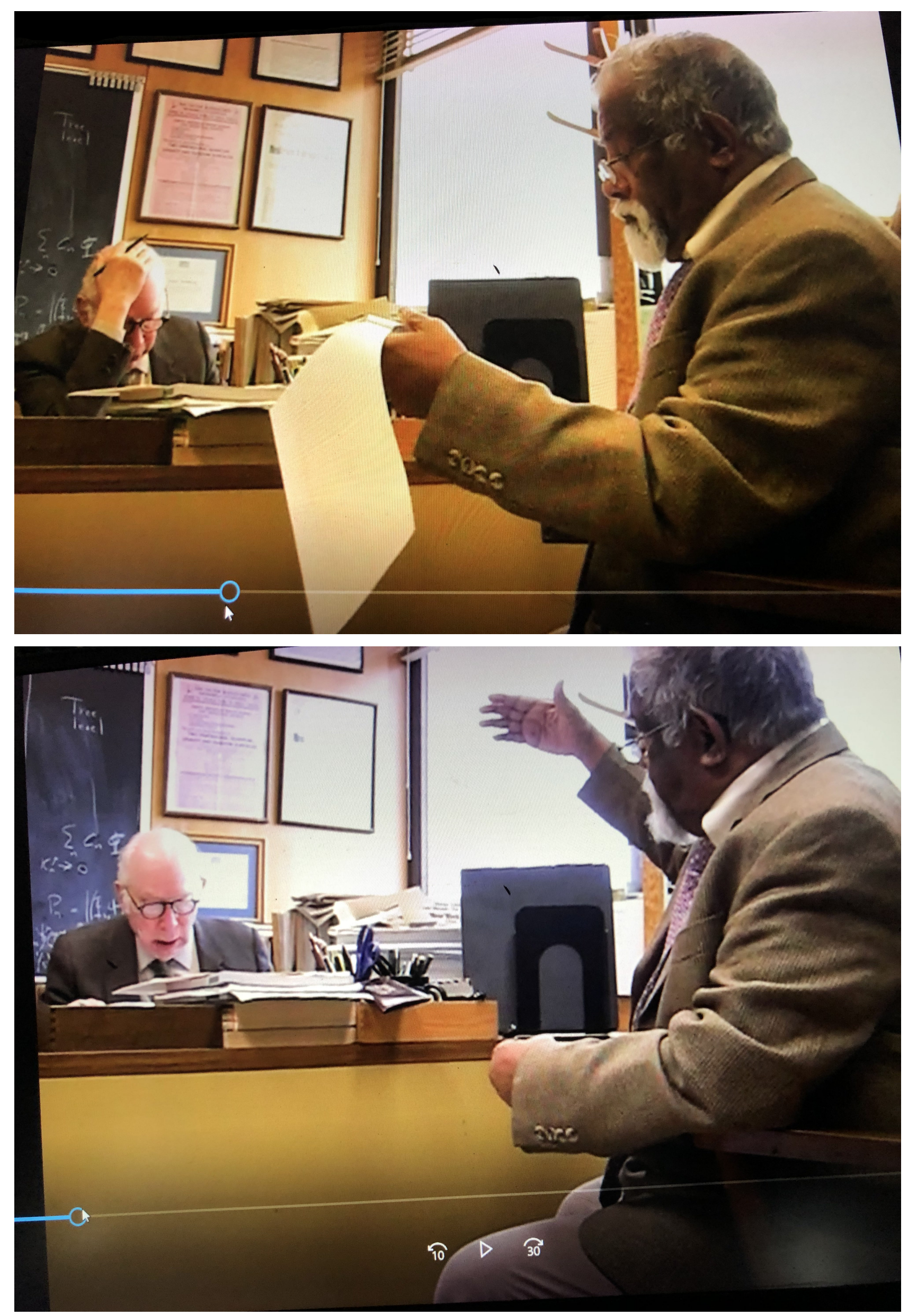

Figure 21: George Sudarshan and Steven Weinberg discussing complete positivity in Weinberg's office at Austin. 


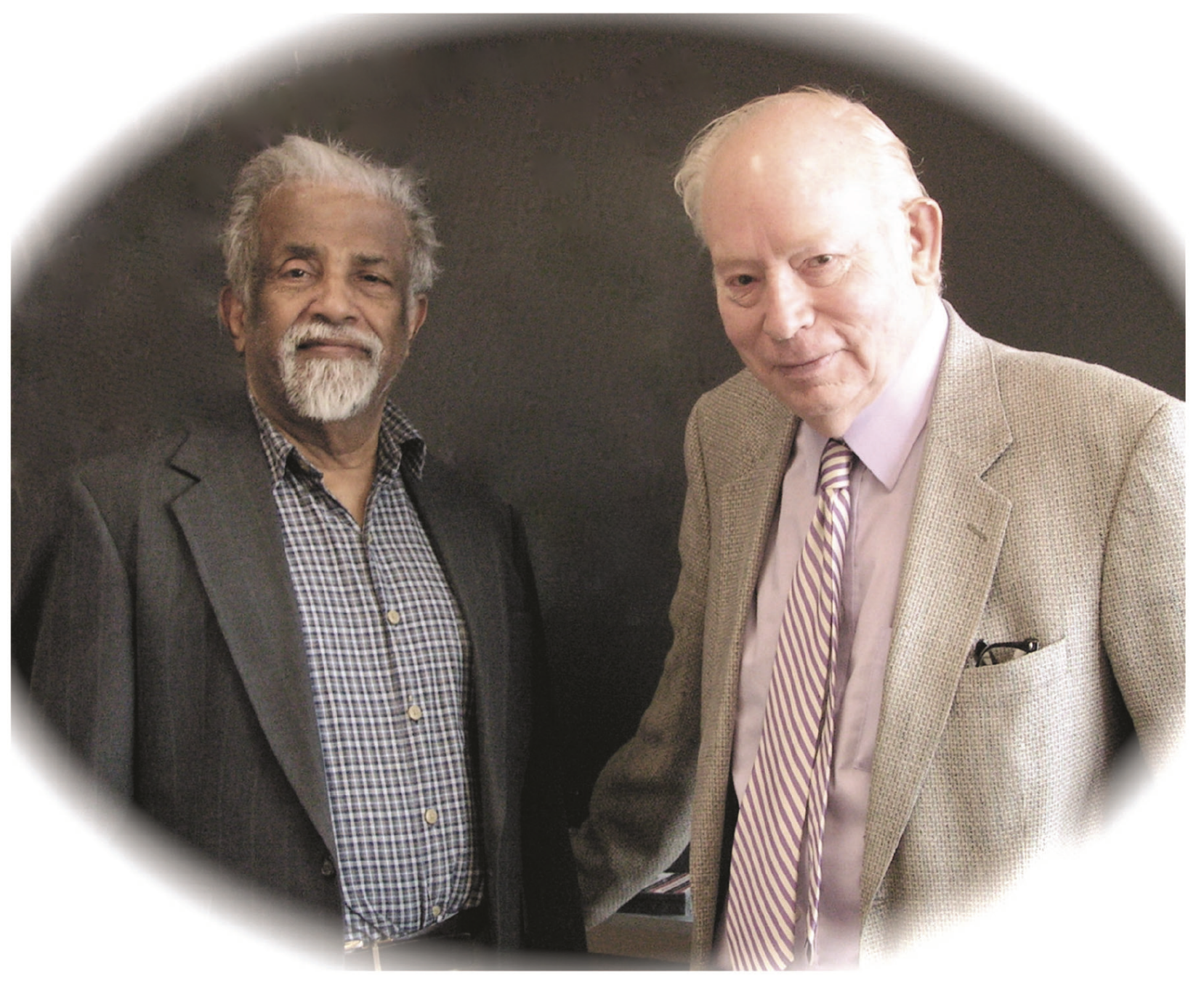

Figure 22: George Sudarshan and Steven Weinberg at Austin. 


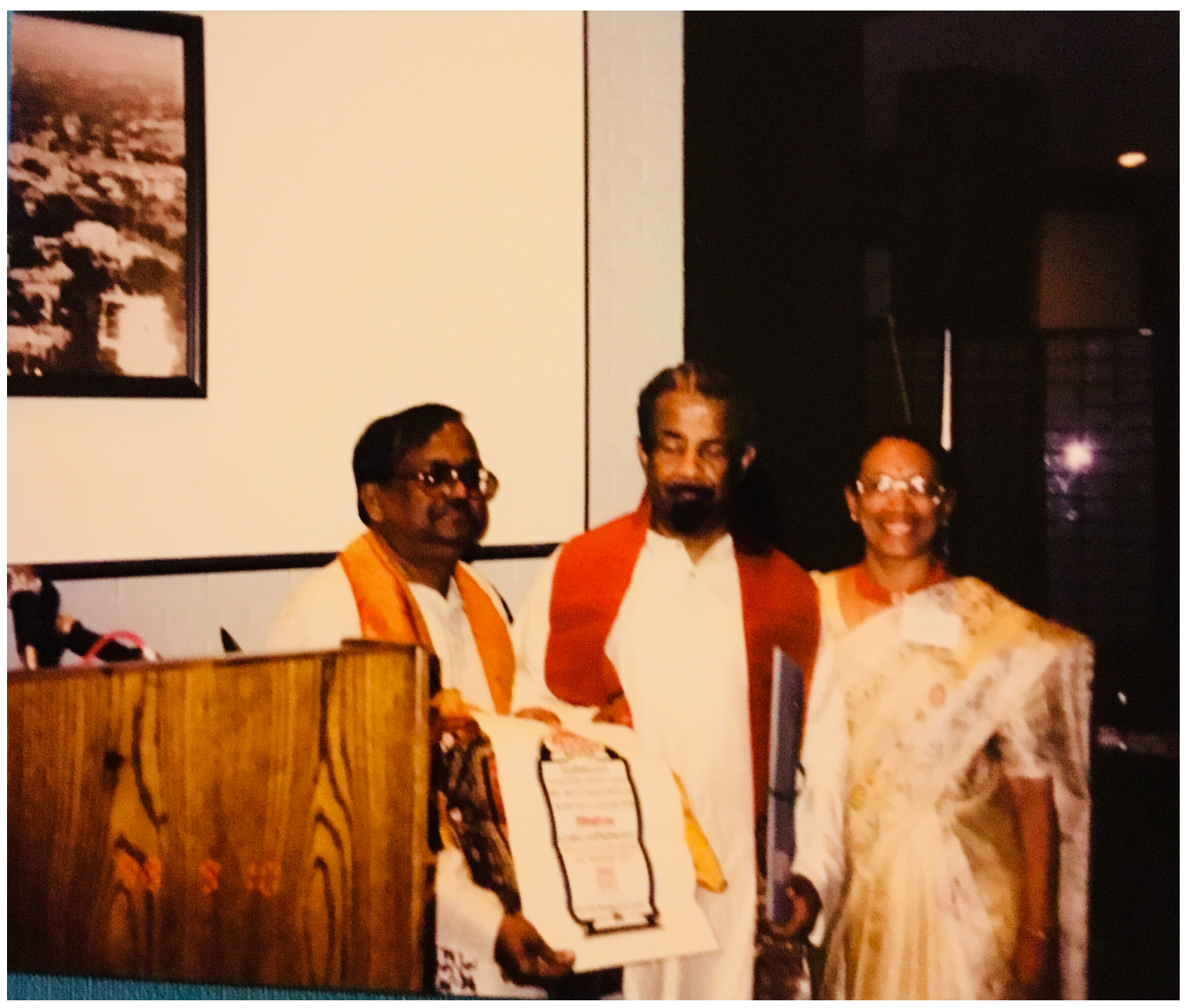

Figure 23: With the Vice Chancellor of Santiniketan and G. Bhamathi receiving the Desikottama award in 1998. 


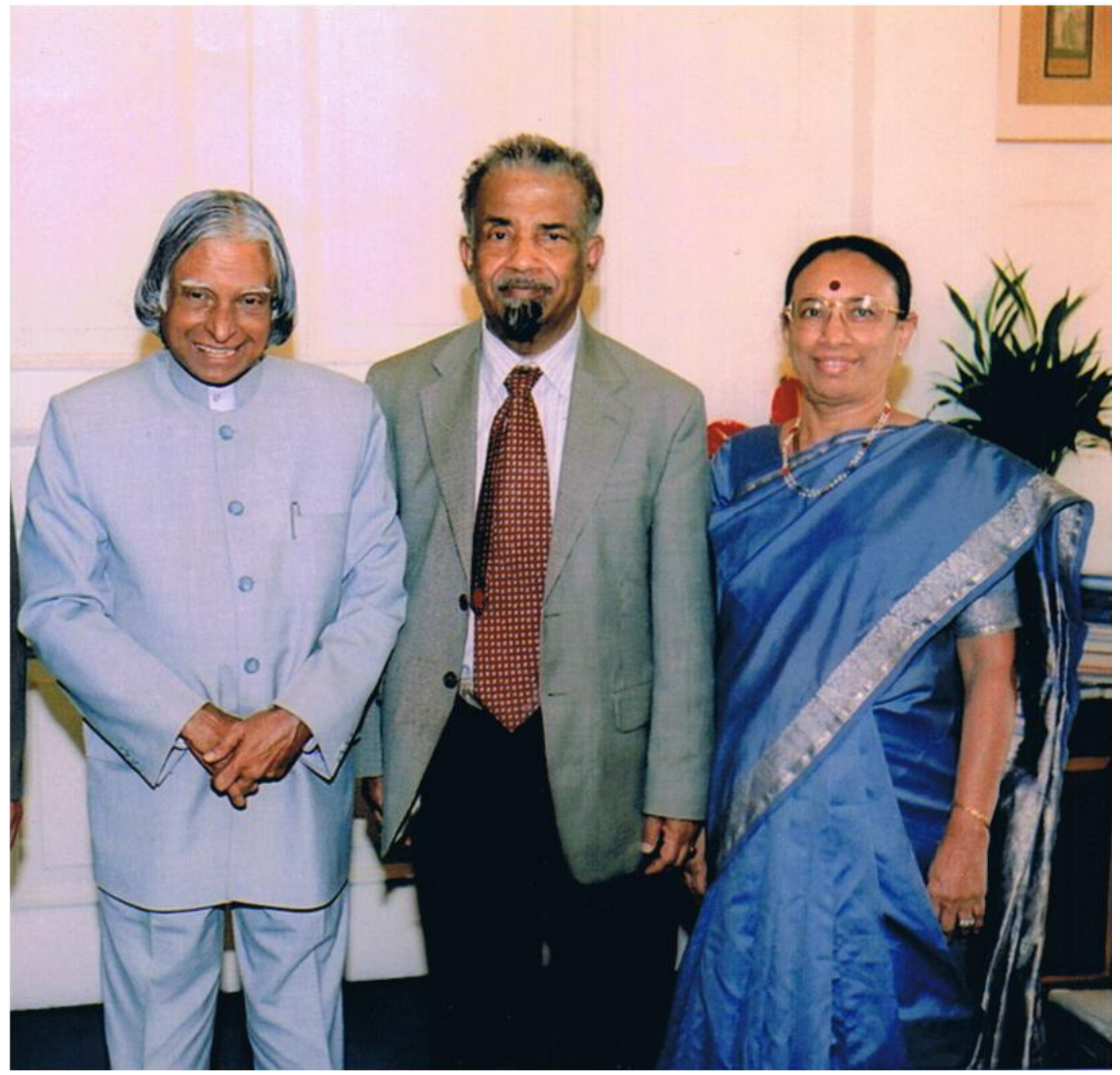

Figure 24: With the former President of India, Dr. A. P. J. Abdul Kalam and G. Bhamathi in 2005. 


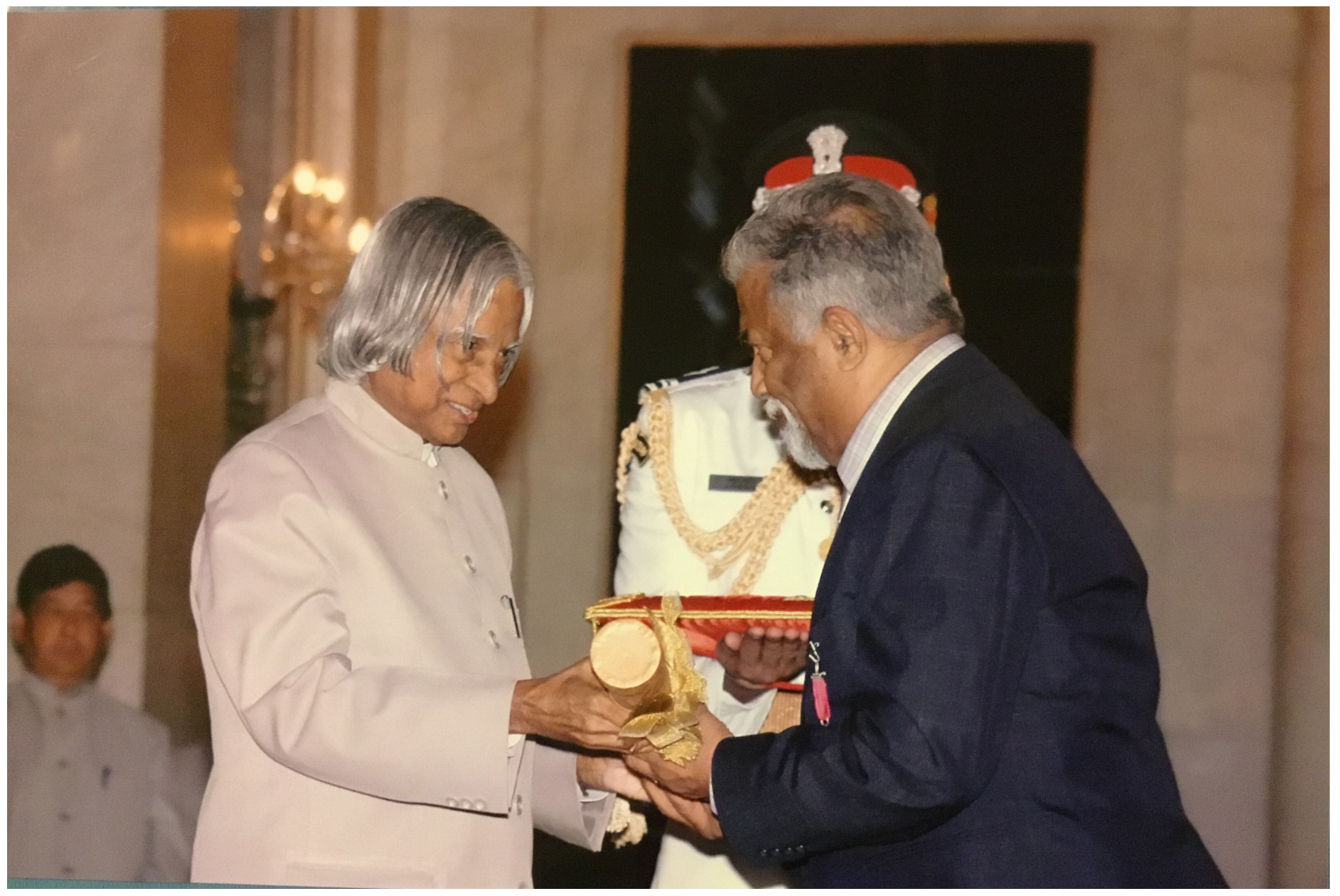

Figure 25: Sudarshan receiving the Padma Vibhushan Award in 2007 from the then President of India, Dr. A. P. J. Kalam. 


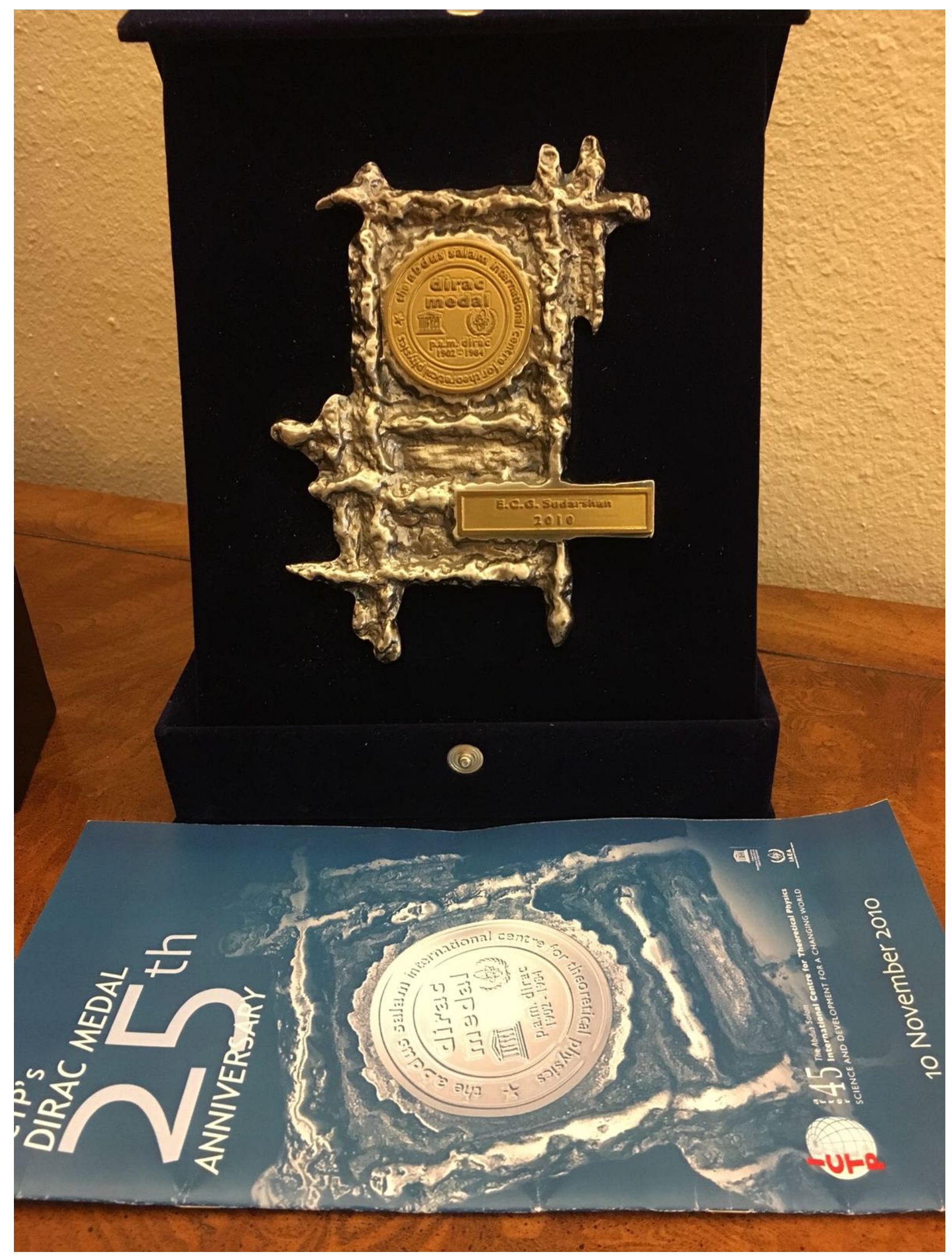

Figure 26: Dirac Medal awarded in 2010. 


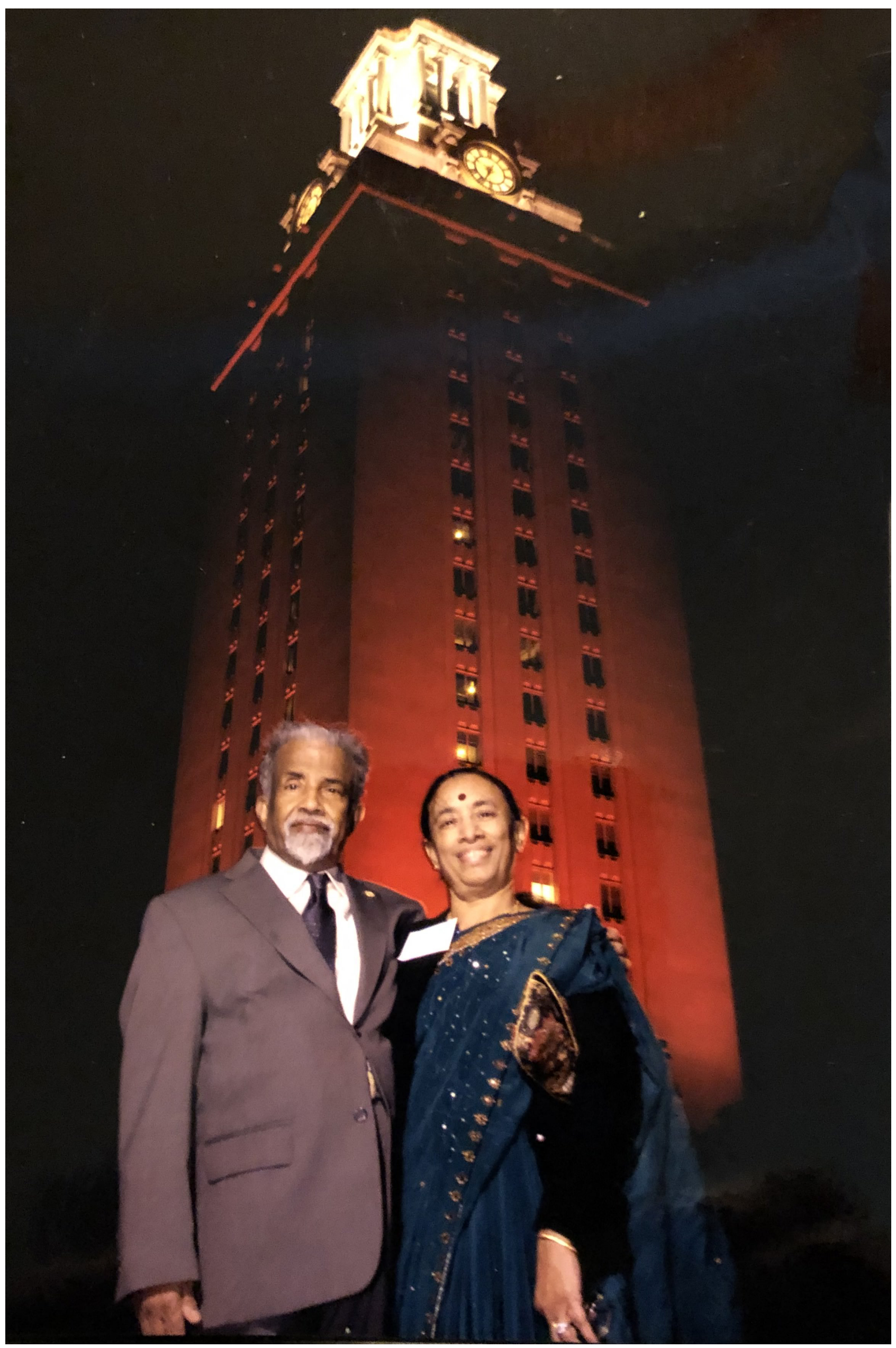

Figure 27: George Sudarshan and G. Bhamathi in front of UT Tower lit in honor of Dirac Medal. 


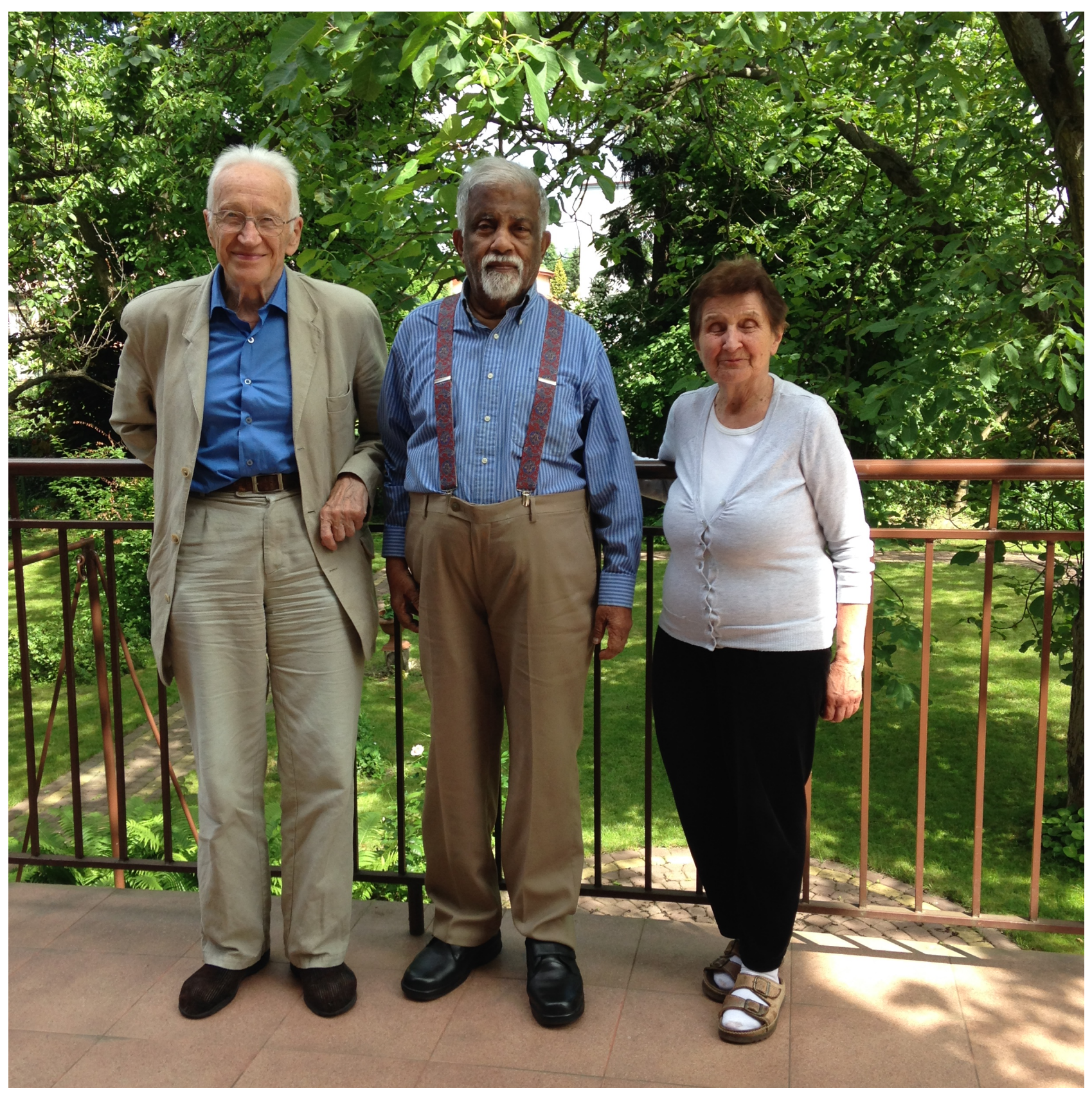

Figure 28: With Białynicki Birula and Zofia Birula in Warsaw 2017. 